\title{
SOME TOPICS CONCERNING HOMEOMORPHIC PARAMETERIZATIONS
}

\author{
Stephen Semmes
}

Abstract

In this survey, we consider several questions pertaining to homeomorphisms, including criteria for their existence in certain circumstances, and obstructions to their existence.

\section{Contents}

1. Wildness and tameness phenomena 4

2. Contractable open sets 8

2.1. Some positive results 11

2.2. Ends of manifolds 16

3. Interlude: looking at infinity, or looking near a point 16

4. Decomposition spaces, $1 \quad 18$

4.1. Cellularity, and the cellularity criterion 24

5. Manifold factors 26

6. Decomposition spaces, $2 \quad 29$

7. Geometric structures for decomposition spaces 32

7.1. A basic class of constructions 32

7.2. Quotient spaces can be topologically standard, but geometrically tricky

7.3. Examples that are even simpler topologically, but still nontrivial geometrically

8. Geometric and analytic results about the existence of good coordinates

8.1. Special coordinates that one might consider in other dimensions

9. Nonlinear similarity: Another class of examples 54

10. Doing pretty well with spaces which may not have nice coordinates

References

The author was partially supported by the National Science Foundation. 


\section{Wildness and tameness phenomena}

Consider the following question. Let $n$ be a positive integer, and let $K$ be a compact subset of $\mathbf{R}^{n}$. If $K$ is homeomorphic to the unit interval $[0,1]$, is there

a global homeomorphism from $\mathbf{R}^{n}$ onto itself

which maps $K$ to a straight line segment?

If $n=1$, then $K$ itself is a closed line segment, and the answer is "yes". When $n=2$, the answer is also "yes", but this is more complicated, and is more in the spirit of the Schönflies theorem in the plane. See [Moi], especially Chapter 10 .

When $n \geq 3$, the answer to the question above can be "no". An $\operatorname{arc} K$ is said to be "tame" (or flat) when a homeomorphism does exist as in (1.1), and "wild" when it does not exist. See [Moi] for some examples of wild $\operatorname{arcs}$ in $\mathbf{R}^{3}$.

Smooth arcs are always tame, as are polygonal arcs, i.e., arcs made up of finitely many straight line segments. For these one can take the corresponding homeomorphism to be smooth or piecewise-linear as well. (Compare with Theorem 1 on p. 134 of [Moi], for instance.) In order for an arc to be wild, some amount of infinite processes are needed.

A simple closed curve in $\mathbf{R}^{3}$ might be smooth or polygonal and still knotted, so that there does not exist a homeomorphism of $\mathbf{R}^{3}$ onto itself which maps the curve onto a standard circle (inside a standard 2-dimensional plane in $\mathbf{R}^{3}$ ). There are many well-known examples of this, like the trefoil knot. Thus, for a closed curve, one defines "wildness" in a slightly differently way, in terms of the existence of local flattenings, for instance. This turns out to be compatible with the case of arcs (for which there is no issue of knottedness), and there are some other natural variants of this.

Here is another basic example, for sets of higher dimension. Suppose that $\gamma$ is a simple closed curve in $\mathbf{R}^{3}$, which is a polygonal curve, and which represents the trefoil knot. Consider the cone over $\gamma$, which gives a 2-dimensional polyhedron in $\mathbf{R}^{4}$, and which is in fact piecewise-linearly equivalent to a standard 2-dimensional cell. One can show that this embedding of the 2-cell is not locally flat at the cone point, i.e., it cannot be straightened out to agree with a standard (geometrically flat) embedding by a homeomorphism defined on a neighborhood in $\mathbf{R}^{4}$ of the cone point. Similar phenomena occur for codimension-2 embeddings in $\mathbf{R}^{n}$ for all $n \geq 4$, as in Example 2.3.2 on p. 59-60 of [Rus1]. 
This phenomenon is special to codimension 2, however. Specifically, a piecewise-linear embedding of a $k$-dimensional piecewise-linear manifold into $\mathbf{R}^{n}$ is locally topologically flat if $n-k \neq 2$ (or if $k=1$ and $n=3$, as before). See Theorem 1.7.2 on p. 34 of [Rus1].

In the context of piecewise-linear embeddings, one can also look for local flattenings which are piecewise-linear. A similar remark applies to other categories of mappings. We shall not pursue this here.

Wild embeddings of cells and spheres (and other manifolds) exist in $\mathbf{R}^{n}$ for all $n \geq 3$, and for all dimensions of the cells and spheres (from 1 to $n-1)$. This includes embeddings of cells and spheres which are not equivalent to piecewise-linear embeddings in codimension 2. We shall mostly consider here issues of existence of topological flattenings or local flattenings, and embeddings which are not normally given as piecewise-linear.

See $[$ Bin6], [Bur], [BurC], [Can1], [Dave1], [Dave2], [Edw1], [Moi], [Rus1], [Rus2] for more information, and for further references. Let us also mention that embeddings, although wild, may still enjoy substantial good behavior. For instance, they may be bilipschitz, so that distances are not increased or decreased by more than a bounded factor, or quasisymmetric, in the sense of [ $\mathbf{T u k V}]$. Roughly speaking, the latter means that relative distances are not distorted too much, rather than distances themselves, as for a bilipschitz mapping. See [Geh1], [LuuV], [Väi2] for some basic results in these directions.

As another version of wildness for embeddings, imagine that one has a compact set $C$ in some $\mathbf{R}^{n}$, and that $C$ is homeomorphic to the usual middle-thirds Cantor set. Can one move $C$ to a subset of a straight line in $\mathbf{R}^{n}$, through a homeomorphism from $\mathbf{R}^{n}$ onto itself?

When $n=1$ this is automatically true. It is also true when $n=2$; see [Moi], especially Chapter 13. In higher dimensions it is not true in general, as is shown by a famous construction of Antoine ("Antoine's necklaces"). See Chapter 18 of [Moi] and [Bla].

How can one tell when a set is embedded wildly or not? As a simple case, let us consider Cantor sets. If $C$ is a compact subset of $\mathbf{R}^{n}$ which lies in a line and is homeomorphic to the Cantor set, and if $n$ is at least 3, then the complement of $C$ in $\mathbf{R}^{n}$ is simply-connected. This is not hard to see. Basically, if one takes a loop in the complement of $C$ and fills it with a disk in $\mathbf{R}^{n}$, and if that disk happens to run into $C$, then one can make small perturbations of the disk to avoid intersecting $C$.

The complement of $C$ is also simply-connected if there is a global homeomorphism from $\mathbf{R}^{n}$ onto itself which maps $C$ into a line. This is 
merely because the homeomorphism itself permits one to reduce to the previous case.

However, Antoine's necklaces have the property that their complements are not simply-connected. See [Moi], [Bla]. Note that the homology of the complement of a compact set in $\mathbf{R}^{n}$ is controlled through the intrinsic topology of the set itself, as in Alexander duality [Spa]. In particular, while the complement of an Antoine's necklace may not be simply-connected, its 1-dimensional homology does vanish.

Versions of the fundamental group play an important role for wildness and taming in general, and not just for Cantor sets. For this one may not take (or want to take) the fundamental group of the whole complementary set, but look at more localized forms of the fundamental group. A specific and basic version of this is the following. Suppose that $F$ is a closed set inside of some $\mathbf{R}^{n}$. Given a point $p \in F$, and a loop $\gamma$ in $\mathbf{R}^{n} \backslash F$ which lies close to $p$, one would like to know whether it is possible to contract $\gamma$ to a point in $\mathbf{R}^{n} \backslash F$ while staying in a small neighborhood of $p$. This second neighborhood of $p$ might not be quite as small the first one; a precise statement would say that for every $\epsilon>0$ there is a $\delta>0$ so that if $\gamma$ lies in $B(p, \delta) \cap\left(\mathbf{R}^{n} \backslash F\right)$, then $\gamma$ can be contracted to a point in $B(p, \epsilon) \cap\left(\mathbf{R}^{n} \backslash F\right)$.

This type of condition is satisfied by standard embeddings of sets into $\mathbf{R}^{n}$, like Cantor sets, cells, and spheres, at least when the dimension of the set is different from $n-2$. For a point in $\mathbf{R}^{2}$, or a line segment in $\mathbf{R}^{3}$, etc., one would get $\mathbf{Z}$ for the corresponding localized fundamental group of the complement of the set. (In the case of a line segment in $\mathbf{R}^{3}$, one should restrict one's attention to points $p$ in the interior of the segment for this.)

Conversely, there are results which permit one to go backwards, and say that localized fundamental group conditions for the complement like these (localized simple-connectedness conditions in particular) lead to tameness of a given set, or other kind of "standard" (non-wild) behavior. See [Bin5], [Bin6], [Bin8], [Bur], [BurC], [Can1], [Can2], [Dave1], [Dave2], [Edw1], [Moi], [Qui1], [Qui2], [Rus1], [Rus2] for more information about localized fundamental groups and their role in wildness phenomena and taming theorems (and for related matters and further references).

One might wonder why $\pi_{1}$ and localized versions of it play such an important role. Some basic points behind this are as follows. For homology (or cohomology), one often has good information from data in the given situation through standard results in algebraic topology, like duality theorems. In circumstances with suitable simple-connectivity, 
one can pass from information about homology to information about homotopy (in general dimensions), as in the Hurewicz and Whitehead theorems. See [Bre1], [Spa].

Another basic point concerns the effect of stabilization. A wild embedding of a set into some $\mathbf{R}^{n}$ can become tame when viewed as an embedding into an $\mathbf{R}^{m}$ with $m>n(m=n+1$ in particular). The same is true for knotting. For instance, a smooth loop may be knotted in $\mathbf{R}^{3}$, but when viewed as a subset of $\mathbf{R}^{4}$, it is always unknotted. This is easy to see in explicit examples (like a trefoil knot).

For some simple and general results about wild embeddings in $\mathbf{R}^{n}$ becoming tame in a larger $\mathbf{R}^{m}$, see Proposition 4 on p. 84 of [Dave2], and the corollaries on p. 85 of [Dave2]. These involve a famous device of Klee. In concrete examples, one can often see the taming in a largerdimensional space directly, and explicitly. Examples of wild sets are often made with the help of various linkings, or something like that, and in a higher-dimensional space one can disentangle the linked parts. This can be accomplished by taking individual pieces and pulling them into a new dimension, moving them around freely there, and then putting them back into the original $\mathbf{R}^{n}$ in a different way.

This simplifying effect of stabilization also fits with the role of localized versions of fundamental groups indicated before. Let $F$ be a closed set inside of some $\mathbf{R}^{n}$, and imagine that one has a loop $\gamma$ in $\mathbf{R}^{n} \backslash F$ which lies in a small ball centered at a point in $F$. In the condition that was discussed earlier, one would like to contract $\gamma$ to a point in the complement of $F$, while remaining in a small ball. If one thinks of $\gamma$ and $F$ as being also inside $\mathbf{R}^{n+1}$, then it is easy to contract $\gamma$ to a point in the complement of $F$ in $\mathbf{R}^{n+1}$, while remaining in a small ball. Specifically, one can first translate $\gamma$ into a parallel copy of $\mathbf{R}^{n}$ inside of $\mathbf{R}^{n+1}$, i.e., into $\mathbf{R}^{n} \times\{a\}$ for some $a \neq 0$ rather than $\mathbf{R}^{n} \times\{0\}$ in $\mathbf{R}^{n+1}$ (using the obvious identifications). This parallel copy is then disjoint from $F$, and one can contract the loop in a standard way. Note that this argument works independently of the behavior of $F$.

Using taming theorems based on localized fundamental group conditions, and considerations like those in the previous paragraph, one can get stronger results on tameness that occurs from stabilization than the ones on p. 84-85 in [Dave2] mentioned above. More precisely, instead of needing $k$ extra dimensions in some cases, it is enough to go from $\mathbf{R}^{n}$ to $\mathbf{R}^{n+1}$. Compare with the bottom of p. 390 and the top of p. 391 in [Dave1], and the references indicated there. (Compare also with the remarks on p. 452 of [Can1].) 


\section{Contractable open sets}

Fix a positive integer $n$.

If $U$ is a nonempty contractable open subset of $\mathbf{R}^{n}$, is

$U$ necessarily homeomorphic to the open unit ball in $\mathbf{R}^{n}$ ?

For the record, to say that $U$ is contractable means that the identity mapping on $U$ is homotopic to a constant, through (continuous) mappings from $U$ into itself. In particular, the homotopy and homology groups of $U$ (of positive dimension) would then be trivial, just as for an $n$-dimensional ball.

When $n=1$, the answer to the question in (2.1) is "yes". In this case, $U$ is either the whole real line, an open segment in the real line, or an open ray. Each of these is easily seen to be homeomorphic to the interval $(-1,1)$, which is the unit ball in this case.

If $n=2$, then the answer to the question in (2.1) is "yes" again. This is a well-known fact, and we shall return to it later, in Section 8.

Starting in dimension 3, the answer to the question in (2.1) is "no". We shall say something about examples for this in a moment, but let us first ask ourselves the following: how might one be able to tell that a given contractable open set in $\mathbf{R}^{n}$ is not homeomorphic to an $n$-dimensional ball?

Here again a localized version of the fundamental group is important. If $n \geq 3$, then a necessary condition for a set $U$ to be homeomorphic to an $n$-dimensional ball is that $U$ be "simply connected at infinity". Roughly speaking, this means that if one takes a closed loop $\gamma$ out near infinity in $U$, then it should be possible to contract $\gamma$ to a point, while staying out near infinity too (although perhaps not as much as $\gamma$ itself is).

Here is a more formal definition. For this we also include "connectedness at infinity" as a first part.

Definition 2.2. Let $U$ be an open set in $\mathbf{R}^{n}$, or a topological space more generally. (Normally one might at least ask that $U$ be locally compact.)

$U$ is connected at infinity if for each compact set $K_{0} \subseteq U$ there is a larger compact set $L_{0} \subseteq U$ such that every pair of points in $U \backslash L_{0}$ is contained in a connected set which is itself contained in $U \backslash K_{0}$. (One can define "arcwise connectedness at infinity" in a similar manner. The two notions are equivalent under assumptions of local arcwise connectedness, and for topological manifolds in particular.) 
$U$ is simply-connected at infinity if it is connected at infinity, and if for every compact set $K_{1} \subseteq U$ there is a larger compact set $L_{1} \subseteq U$ so that if $\gamma$ is an arbitrary closed loop in $U \backslash L_{1}$ (i.e., an arbitrary continuous mapping from the unit circle $\mathbf{S}^{1}$ into $U \backslash L_{1}$ ), then $\gamma$ is homotopic to a constant through continuous mappings from the circle into $U \backslash K_{1}$.

If $U$ is the unit ball in $\mathbf{R}^{n}, n \geq 3$, then $U$ is simply-connected at infinity. Indeed, let $B(0, r)$ denote the open ball in $\mathbf{R}^{n}$ with center 0 and radius $r$, and let $\bar{B}(0, r)$ denote the corresponding closed ball. Then every compact subset of $B(0,1)$ is contained in $\bar{B}(0, r)$ for some $r<1$. For each $r<1, \bar{B}(0, r)$ is a compact subset of $B(0,1)$, and $B(0,1) \backslash \bar{B}(0, r)$ is connected when $n \geq 2$, and simply-connected when $n \geq 3$. This is because $B(0,1) \backslash \bar{B}(0, r)$ is homeomorphic to $\mathbf{S}^{n-1} \times(r, 1)$, and $\mathbf{S}^{j}$ is connected when $j \geq 1$, and simply-connected when $j \geq 2$.

The property of being simply-connected at infinity is clearly preserved by homeomorphisms. Thus to get a contractable open set $U$ in $\mathbf{R}^{n}$ which is not homeomorphic to an $n$-dimensional ball, it suffices to choose $U$ so that it is not simply-connected at infinity.

If $U$ is contractable, then it is simply-connected itself in particular. If $U$ is simply-connected, connected at infinity, and not simply-connected at infinity, then it means that there is a compact set $K \subseteq U$ and loops $\gamma$ in $U$ which lie as far towards infinity as one would like (i.e., in the complement of any given compact subset of $U$ ) such that (a) $\gamma$ can be contracted to a point in $U$, and (b) $\gamma$ cannot be contracted to a point in $U \backslash K$. To put it another way, these loops $\gamma$ can be contracted to points in $U$, but in doing this one always has to pass through at least one element of the compact set $K$.

A mechanism for having this happen for a set $U$ contained in $\mathbf{R}^{3}$ is given by the construction of "the Whitehead continuum" [Whit]. (See also [Dave2], [Kir].) Here is an outline of the procedure.

Start with a standard smooth "round" solid torus $T$ in $\mathbf{R}^{3}$. Here $T$ should be a compact set, i.e., it should contain its boundary.

Next one chooses another smooth solid torus $T_{1}$ inside $T$. More precisely, $T_{1}$ should lie in the interior of $T$. One chooses $T_{1}$ in a particular way, which can be imagined as follows. (Pictures can be found on p. 68 of [Dave2] and p. 82 of [Kir].) First take a "small" solid torus in $T$, small enough to be contained in a topological ball in $T$. One can think of grabbing hold of this small solid torus at two ends, and then stretching them around the "hole" in the larger torus $T$. One stretches them around the two different sides of the hole in $T$. To get $T_{1}$, these two ends should hook around each other on the other side of the hole. In other 
words, one might imagine having the two ends of the small solid torus from before, stretched around opposite sides of $T$, and then passing one across the other, until they do not touch any more, but are clasped together, like two hooks, or two links in a chain. The configuration looks locally like two hooks or links clasped together, but in fact one has two ends of the single solid torus $T_{1}$, wrapped around the hole in $T$.

If $T_{1}$ is chosen in this way, then it has the following two basic properties. The first is that it is homotopically trivial in $T$. That is, the identity mapping on $T_{1}$ is homotopic to a constant mapping through (continuous) mappings from $T_{1}$ into $T$. This follows exactly the description above; in making the homotopy, one is allowed to stretch or move $T_{1}$ around as much as one like, and one is allowed to have different parts of (images of) $T_{1}$ cross each other in $T$. To put it a bit differently, the mappings being deformed are not required to be injective.

The second property is that $T_{1}$ is not "isotopically trivial". This means that one cannot continuously deform $T_{1}$ through an isotopy of $T$ into a set which lies in a ball contained in $T$. In effect, this means that one cannot continuously deform $T_{1}$ inside $T$ in such a way that $T_{1}$ ends up in a ball in $T$, and so that the deformations do not ever cross each other (unlike the homotopy in the previous paragraph). If one could get $T_{1}$ inside a ball in $T$, then one could continue the deformation to get an isotopy into an arbitrarily small ball. One would not ask for shrinking $T_{1}$ to a point here, because this is automatically prevented by injectivity (independent of clasping or not).

This explains how $T$ and $T_{1}$ should be chosen. Since $T_{1}$ is a 3 -dimensional smooth solid torus in its own right, one can repeat the process to get another smooth solid torus $T_{2}$ contained in it, and in fact contained in the interior of $T_{1}$. In other words, since $T$ and $T_{1}$ are both smooth solid tori, they are diffeomorphic to each other in particular, and this can be used to make precise the idea of "repeating the process". Specifically, if $\phi: T \rightarrow T_{1}$ is such a diffeomorphism, then one can take $T_{2}$ to be $\phi\left(T_{1}\right)$.

One then repeats the process indefinitely, getting smooth solid tori $T_{j}$ for $j=1,2, \ldots$ such that $T_{j+1}$ is contained in the interior of $T_{j}$ for each $j$, and so that $T_{j+1}$ is arranged in $T_{j}$ in the same way as $T_{1}$ is arranged in $T$.

Now let $W$ be the intersection of all these solid tori $T_{j}$. This gives a nonempty compact set in $\mathbf{R}^{3}$. We can think of $W$ as lying inside of $\mathbf{S}^{3}$, and then take $U=\mathbf{S}^{3} \backslash W$. One can also rotate this around so that $U$ actually lies in $\mathbf{R}^{3}$. One can show that $U$ is contractable, but not simplyconnected at infinity. See [Dave2], [Kir], [Whit] for more information. (For the purposes of looking at $U$, the complement of $W$, it can be 
convenient to use a modestly different description of the construction, in which one builds $U$ up from smaller pieces in an "increasing" manner, analogous to the "decreasing" construction for $W$ above.)

Although $U$ is not homeomorphic to a 3-dimensional ball in this case, the Cartesian product of $U$ with a nonempty open interval is homeomorphic to a 4-dimensional ball. This is attributed to Arnold Shapiro in [Bin3]; see also Section 10 of [Bin4] and [Kir]. This is analogous to the effect of stabilization before, in Section 1. In particular, one can check directly that taking the Cartesian product with the interval gets rid of the problem that $U$ itself has with simple-connectivity at infinity.

Beginning in dimension 4, there are contractable open sets in $\mathbf{R}^{n}$ which are not topological $n$-balls, and which have the additional feature that their closures are compact manifolds with boundary. This last does not work in dimension 3 , and, for that matter, the complement of the Whitehead continuum in $\mathbf{S}^{3}$ cannot be realized as the interior of a compact manifold with boundary, whether or not this compact manifold should occur as the closure of the set in $\mathbf{S}^{3}$. The reason is that if such a compact manifold did exist, its boundary would be a 2-dimensional surface with the homology of the 2 -sphere. We shall say more about this in a moment. In this case the boundary would have to be homeomorphic to the 2 -sphere. This would contradict the failure of simple-connectivity at infinity for the original space, since $\mathbf{S}^{2}$ is simply-connected.

The difference with $n \geq 4$ is that the boundary can be a homology $(n-1)$-sphere (i.e., a manifold with the same homology as $\left.\mathbf{S}^{n-1}\right)$ which is not simply-connected. The interior then fails to be simply-connected at infinity again, and is not homeomorphic to an $n$-ball in particular.

For some related information and references concerning these examples in dimensions greater than or equal to 4 , see [Dave2], including the top of p. 94, and the discussion on p. 103-104.

\subsection{Some positive results.}

For dimensions $n \geq 4$, it is known that every contractable topological manifold $M$ which is simply-connected at infinity is homeomorphic to $\mathbf{R}^{n}$. See [Sta] for $n \geq 5$, and Corollary 1.2 on p. 366 of [Fre] for $n=4$. A related reference is $[\mathbf{M c M Z}]$. Actually, [Sta] is stated for the piecewise-linear category; one can go from there to the topological category via $[\mathbf{K i r S}]$. The four-dimensional result does not work in the smooth or piecewise-linear categories (which are equivalent in dimension 4), because of the existence of "fake $\mathbf{R}^{4}$ 's" (smooth manifolds homeomorphic to $\mathbf{R}^{4}$, but not diffeomorphic to it). Concerning the latter, see [FreQ] (p. 122 in particular) and [Kir] (Chapter XIV). 
These topics are also related to "McMillan's cellularity criterion", in $[\mathbf{M c M}]$. A 4-dimensional version of this is given in [Fre], in Theorem 1.11 on p. 373. We shall discuss cellularity and this criterion further in Subsection 4.1.

Now let us look more closely at the case of compact manifolds with boundary. Suppose that $N$ is an $n$-dimensional compact topological manifold with boundary. Consider the following question:

If $N$ is contractable and $\partial N$ is a topological $(n-1)$-sphere, is $N$ homeomorphic to the closed unit ball in $\mathbf{R}^{n}$ ?

This question is actually equivalent to the Poincaré conjecture (in dimension $n$, and in the topological category). This is a well-known fact. The main points are the following. If one is given a compact $n$-dimensional topological manifold without boundary which is a homotopy $n$-sphere, then one can get an $n$-dimensional manifold $N$ as in (2.3) from it by cutting out a topological ball (with tame boundary). If this manifold $N$ is homeomorphic to the closed unit ball in $\mathbf{R}^{n}$, then one can obtain that the original space was homeomorphic to the standard $n$-sphere, by gluing the ball which was removed back in. (We shall say more about this in Remark 2.4.) Conversely, given a manifold $N$ as in (2.3), one can get a homotopy $n$-sphere from it by gluing in a ball along the boundary of $N$. To go from this and the Poincaré conjecture to the conclusion that $N$ is homeomorphic to a closed ball, one can use the "generalized Schönflies theorem", discussed later in this section (after Remark 2.4).

In particular, the answer to (2.3) is known to be "yes" when $n \neq 3$, and the problem is open for $n=3$.

There are some analogous relationships between the Poincaré conjecture and contractable open manifolds. Namely, if one starts with a compact $n$-dimensional topological manifold without boundary $P$ which is a homotopy $n$-sphere, then one can get an $n$-dimensional contractable open manifold by removing a point $x$ from $P$. If $n \geq 3$, then $P \backslash\{x\}$ will also be simply-connected at infinity, as one can check using the manifold structure of $P$ around $x$. If one knows that $P \backslash\{x\}$ is homeomorphic to $\mathbf{R}^{n}$, then one can deduce that $P$, which is topologically the same as the one-point compactification of $P \backslash\{x\}$, is homeomorphic to $\mathbf{S}^{n}$.

However, it is not as easy to go in the other direction, from contractable open manifolds which are simply-connected at infinity to compact manifolds which are homotopy-equivalent to a sphere, as it is in (2.3). One can take the one-point compactification of the open manifold to get a compact space, but it is not immediately clear that this space 
is a manifold. The simple-connectivity at infinity for the open manifold is a necessary condition for this (when $n \geq 3$ ), but the converse is more complicated. There are broader issues concerning the behavior of open manifolds at infinity, and we shall mention some aspects of this in Subsection 2.2 and Section 3.

For the first part, about going from $P$ to an open manifold, suppose that one is in a situation where there is a general result to the effect that an $n$-dimensional contractable open manifold which is simply-connected at infinity is homeomorphic to $\mathbf{R}^{n}$ for some fixed $n$. As above, one can use this to show that a compact $n$-dimensional manifold $P$ (without boundary) which is homotopy-equivalent to $\mathbf{S}^{n}$ is homeomorphic to $\mathbf{S}^{n}$. A complication with this type of argument is that one does not necessarily say too much about the behavior of the homeomorphism at the point $x$ which was removed and added back again (in the notation before), even if one knows more about $P$ and the homeomorphism between $P \backslash\{x\}$ and $\mathbf{R}^{n}$. In this respect, arguments that go through compact manifolds with boundary, as in (2.3), can work better; there are also some tricky aspects in this case, though, and we shall say more about this next.

Remark 2.4. There are some subtleties about gluing in balls in the context of (2.3) and its correspondence with the Poincaré conjecture in the smooth category. If one takes two copies of the closed unit ball in $\mathbf{R}^{n}$, and glues them together using a homeomorphism between their boundaries, then the resulting space is homeomorphic to a standard $n$-dimensional sphere. This is a standard observation (which can be proved using the fact mentioned in the next paragraph), and it works for any gluing homeomorphism. If the gluing map is a diffeomorphism, then the resulting space is a smooth manifold in a natural way, but it may not be diffeomorphic to a standard sphere. Exotic spheres can be viewed in this manner, as gluings of standard closed balls through (tricky) diffeomorphisms along their boundaries.

In the topological case, one can use the following fact. Let $B_{n}$ denote the closed unit ball in $\mathbf{R}^{n}$. If $h$ is a homeomorphism from $\partial B_{n}$ onto itself, then $h$ can be extended to a homeomorphism from $B_{n}$ onto itself. One can do this by a straightforward "radial extension". This method also works for the analogous statement in the piecewise-linear category. However, in the smooth category, a radial extension like this is not smooth in general at the origin in $B_{n}$. An extension to a diffeomorphism may simply not exist (radial or not). 
In any of the three categories, once one has an extension like this, one can use it to get an equivalence between the space obtained by gluing together the two copies of $B_{n}$, and the standard $n$-dimensional sphere. The extension unwinds the effect of the gluing map, if the gluing map is not the standard one. In the smooth case, this may not be possible, and this occurs with exotic spheres.

Let us look some more at (2.3), in the topological category. If $N$ happens to be given as a subset of $\mathbf{R}^{n}$, in addition to the conditions in (2.3), then $N$ is homeomorphic to the closed unit ball in $\mathbf{R}^{n}$. This can be derived from the "generalized Schönflies theorem" [Brow1], [Maz], [Mors]. This result says that if one has an embedding $f$ of $\mathbf{S}^{n-1} \times[-1,1]$ into $\mathbf{R}^{n}$, then $f\left(\mathbf{S}^{n-1} \times\{0\}\right)$ can be realized as the image of $\mathbf{S}^{n-1}$ under a homeomorphism mapping all of $\mathbf{R}^{n}$ onto itself. See also Theorem 6 on p. 38 of [Dave2].

Let us be a bit more precise about the way that the generalized Schönflies theorem is used here. The first point is that the boundary of $N$ is "collared" in $N$. This means that there is a neighborhood of $\partial N$ in $N$ which is homeomorphic to $\partial N \times[0,1)$, and where the homeomorphism maps each point $z \in N$ to $(z, 0) \in \partial N \times\{0\}$. The assumption that $N$ be a topological manifold with boundary gives a local version of this at each point in $\partial N$, and one can derive the existence of a global collaring from a result of Brown. See [Brow2], [Con] and Theorem 8 on p. 40 of [Dave2].

On the other hand, to apply the generalized Schönflies theorem, one needs a topological ( $n-1)$-sphere in $\mathbf{R}^{n}$ which is "bicollared", i.e., occurs as $f\left(\mathbf{S}^{n-1} \times\{0\}\right)$ for some embedding $f: \mathbf{S}^{n-1} \times[-1,1] \rightarrow \mathbf{R}^{n}$. The boundary of $N$ is collared inside of $N$, but may not be bi-collared inside $\mathbf{R}^{n}$. To deal with this, one can use a parallel copy of $\partial N$ in the interior of $N$, provided by the collaring of $\partial N$ inside $N$. This parallel copy is now bi-collared in the interior of $N$, because of the collaring that we have for $N$.

If $N$ lies inside $\mathbf{R}^{n}$, then this parallel copy is also bi-collared inside $\mathbf{R}^{n}$. One can apply the generalized Schönflies theorem, to get that the region in $\mathbf{R}^{n}$ bounded by this parallel copy of $\partial N$, together with this copy of $\partial N$ itself, is homeomorphic to the closed unit ball in $\mathbf{R}^{n}$. To get back to $N$ in its entirety, one uses the original collaring of $\partial N$ inside $N$, to know that the missing part of $N$ is homeomorphic to the product of $\partial N \cong \mathbf{S}^{n-1}$ with an interval, and to glue this to the other piece without causing trouble. 
Thus one can get a positive answer to (2.3) when $N$ lies inside $\mathbf{R}^{n}$, using the generalized Schönflies theorem. This is simpler than the solutions of the Poincaré conjecture, and it does not require any restrictions on the dimension $n$. The assumption of contractability of $N$ is not needed for this either. (For arbitrary manifolds, not necessarily embedded in $\mathbf{R}^{n}$, this assumption would be crucial.)

In general, if $N$ is an $n$-dimensional compact topological manifold with boundary which is contractable, then the boundary $\partial N$ is always a homology sphere (has the same homology groups as an $(n-1)$-dimensional sphere). This is a well-known fact. One could use Theorem 9.2 on p. 357 of [Bre1], for instance. Conversely, any compact $(n-1)$-dimensional topological manifold without boundary which is a homology sphere can be realized as the boundary of an $n$-dimensional compact topological manifold with boundary which is contractable. This is elementary for $n \leq 3$, where the homology spheres are all ordinary spheres, and can be filled with balls. For $n \geq 5$, this is given in [Ker1], in the piecewise-linear category (for both the homology sphere and its filling by a contractable manifold). For $n \geq 6$, one can convert this into a statement about topological manifolds, through the Kirby-Siebenmann theory [KirS]. See also the bottom of p. 184 of [Dave2]. For $n=5$ in the topological category, see the corollary on p. 197 of [FreQ]. See also Corollary 2B on p. 287 of [Dave2] for $n \geq 5$ and the topological category. (For the smooth category in high dimensions, there are complications which come from the existence of exotic spheres, as in the discovery of Milnor.)

For $n=4$, see [Fre], [FreQ]. In particular, see Theorem 1.4' on p. 367 of [Fre], and Corollary $9.3 \mathrm{C}$ on p. 146 of [FreQ]. In this case it can happen that the filling by a contractable manifold cannot be given as a piecewise-linear manifold. The boundary $\partial N$ would always admit a unique piecewise-linear structure, by well-known results about 3-dimensional manifolds (as in [Moi]). Concerning the possible lack of piecewise-linear filling for a homology 3-sphere by a contractable 4-manifold, see [Fre], [FreQ], [Kir].

A famous example of a homology 3 -sphere which is not simply-connected is given by the "Poincaré homology sphere". This is a quotient of the standard $\mathbf{S}^{3}$ by the (finite) icosahedral group. See Theorem 8.10 on p. 353 of [Bre1]. This is a particular example where a contractable filling exists among topological 4-manifolds, but not among piecewiselinear manifolds. See [Fre], [FreQ], [Kir].

If $H$ is a $k$-dimensional compact manifold (without boundary) which is $k$-dimensional homology sphere, and if $H$ is also simply-connected, then 
$H$ is homotopy-equivalent to the standard $k$-dimensional sphere. This is a standard fact from topology. In this case, the Poincaré conjecture in dimension $k$ would seek to say that $H$ should be homeomorphic to $\mathbf{S}^{k}$.

Note that if $N$ is a compact manifold with boundary, then $\partial N$ is simply-connected if and only if the interior of $N$ is simply-connected at infinity in the sense of Definition 2.2.

\subsection{Ends of manifolds.}

Suppose that $M$ is an $n$-dimensional manifold without boundary which is "open", i.e., not compact. What can one say about the "ends" of $M$ ?

In particular, when can $M$ be realized as the interior of a compact manifold with boundary? This would be a nice way of "taming" the end.

This type of issue is clearly related to the questions considered throughout this section. It also makes sense in general, whether or not $M$ is contractable, or one expects it to be homeomorphic to a ball, or one expects the end to be cylindrical.

Some sufficient conditions for realizing an open manifold as the interior of a compact manifold with boundary in high dimensions are given in $[\mathbf{B r o L L}]$. A characterization for this is given in [Sie]. See also [Ker2] concerning the latter.

For dimension 5 (with 4-dimensional boundaries), see [Qui3] and Section 11.9 of $[$ FreS]. For dimension 4 (with 3-dimensional boundaries), see Theorem 1.12 on p. 373 of [Fre], and Section 11.9 in [FreQ]. Concerning dimension 3, see p. 216 of [FreQ].

In all of these, the fundamental group at infinity plays an important role.

\section{Interlude: looking at infinity, or looking near a point}

Let $M$ be a topological manifold of dimension $n$, and without boundary. Assume that $M$ is open, i.e., not compact.

Define $\widehat{M}$ to be the one-point compactification of $M$, through the usual recipe. That is, one adds to $M$ a special point $q$, the point at infinity, and the neighborhoods of $q$ in $\widehat{M}$ are given by sets of the form $\widehat{M} \backslash K$, where $K$ is a compact subset of $M$.

Consider the following question:

(3.1) Under what conditions is $\widehat{M}$ a topological manifold?

One might look at this as a kind of local question, about the behavior of a space at a given point, or as a question about large-scale behavior 
of $M$. It is not hard to see that $\widehat{M}$ will be a topological manifold exactly when $M$ looks like (is homeomorphic to) $\mathbf{S}^{n-1} \times[0,1)$ outside of a set with compact closure. Equivalently, $\widehat{M}$ is a manifold exactly when $M$ can be realized as the interior of a compact manifold with boundary, where the boundary is homeomorphic to $\mathbf{S}^{n-1}$. (This uses Brown's theorem about the existence of collars for boundaries of manifolds with boundary, as in [Brow2], [Con] and Theorem 8 on p. 40 of [Dave2].)

The large-scale perspective of (3.1) is somewhat close in outlook to Section 2, especially Subsection 2.1, while the local view is perhaps more like the perspective in Section 1. Concerning the latter, one might think of local taming properties of embedded sets in terms of existence of "normal bundles" for the embedded sets. Similarly, one can think of (3.1) as asking about the existence of a normal bundle for $\widehat{M}$ at the point $q$. In this regard, one might compare with the discussion in Section 9.3 in [FreQ], especially Theorem 9.3A and Corollary 9.3B.

For the record, let us note that a necessary condition for $\widehat{M}$ to be a manifold is that

$M$ is simply-connected at infinity,

at least if $n \geq 3$. In "local" language, we can reformulate this condition as follows: for every neighborhood $U$ of $q$ in $\widehat{M}$, there is a neighborhood $V$ of $q$ such that $V \subseteq U$,

$$
\begin{aligned}
& \text { every pair of points } x, y \in V \backslash\{q\} \\
& \text { lies in a connected set in } U \backslash\{q\},
\end{aligned}
$$

and

(3.4) every loop $\gamma$ in $V \backslash\{q\}$ can be contracted to a point in $U \backslash\{q\}$.

This is similar to the localized fundamental group conditions mentioned in Section 1.

Let us now think of $\widehat{M}$ as being any topological space, and not necessarily the one-point compactification of an open manifold. For a given point $q \in \widehat{M}$, one can still ask whether $\widehat{M}$ is an $n$-dimensional manifold at $q$, i.e., if there is a neighborhood of $q$ in $\widehat{M}$ which is homeomorphic to an open ball in $\mathbf{R}^{n}$. The necessary condition in the preceding paragraph still applies (when $n \geq 3$ ), concerning local simple-connectivity of $\widehat{M} \backslash\{q\}$ near $q$ (as in (3.3) and (3.4)).

For the rest of this section, let us assume that $n \geq 3$. Note that there are special results for detecting manifold behavior in a space of dimension 1 or 2 . This is reviewed in the introduction of [Fer]. 
As a special case, imagine now that $\widehat{M}$ is a finite polyhedron of dimension $n$. Let $L$ denote the codimension- 1 link of $q$ in $\widehat{M}$. Thus $L$ is an $(n-1)$-dimensional finite polyhedron, and $\widehat{M}$ looks locally at $q$ like a cone over $L$.

In order for $\widehat{M}$ to be an $n$-dimensional topological manifold in a neighborhood of $q$, the link $L$ should be fairly close to a standard ( $n-$ 1 )-sphere. In particular, it is not hard to see that $L$ should be homotopyequivalent to $\mathbf{S}^{n-1}$. This implies that $L$ should be connected and simplyconnected, under our assumption that $n$ is at least 3 .

In fact, in the case where $\widehat{M}$ is a finite polyhedron, the connectedness and simple-connectedness of the link $L$ around $q$ are equivalent to the local connectivity and simple-connectivity conditions for $\widehat{M} \backslash\{q\}$ near $q$ indicated above, with (3.3) and (3.4). This is not hard to see, and it is also rather nice. To put it a bit differently, imagine that one starts with the class of finite polyhedra, and then tries to go to more general contexts of topological spaces. The local connectivity and simple-connectivity conditions for $\widehat{M} \backslash\{q\}$ at $q$ as described above provide a way to capture the information in the connectedness and simple-connectedness of the codimension-1 link at $q$ in the case where $\widehat{M}$ is a polyhedron, in a manner that makes sense for arbitrary topological spaces, without special structure as one has for finite polyhedra.

\section{Decomposition spaces, 1}

Let $n$ be a positive integer, and let $K$ be a nonempty compact subset of $\mathbf{R}^{n}$. One could also consider general manifolds instead of $\mathbf{R}^{n}$ here, but we shall generally stick to Euclidean spaces for simplicity. The main ideas come up in this case anyway.

Imagine shrinking $K$ to a single point, while leaving the rest of $\mathbf{R}^{n}$ alone, and looking at the topological space that results. This can be defined more formally as follows. Let us write $\mathbf{R}^{n} / K$ for the set which consists of the points in $\mathbf{R}^{n}$ which do not lie in $K$, together with a single point which corresponds to $K$ itself. In other words, this is where we shrink $K$ to a single point. This set can be given a topology in a standard way, so that a subset $U$ of $\mathbf{R}^{n} / K$ is open if and only if its inverse image back in $\mathbf{R}^{n}$ is open. Here "inverse image" uses the automatic quotient mapping $\mathbf{R}^{n}$ to $\mathbf{R}^{n} / K$. (In concrete terms, the inverse image of $U$ in $\mathbf{R}^{n}$ means the set of points in $\mathbf{R}^{n}$ which correspond to elements of $U$, where one includes all points in $K$ if the element of $\mathbf{R}^{n} / K$ associated to $K$ lies in $U$.) 
This type of quotient $\mathbf{R}^{n} / K$ is a special case of a "decomposition space". We shall discuss the general situation further in Section 6 , but this special case already includes a lot of interesting examples and phenomena.

Now let us consider the following question:

$$
\text { Given } K \text { as above, when is } \mathbf{R}^{n} / K \text { a topological manifold? }
$$

This is really a special case of the situation in Section 3. For this it is better to use $\mathbf{S}^{n}$ instead of $\mathbf{R}^{n}$, so that $\mathbf{S}^{n} / K$-defined in the same manner as above - is equivalent to the one-point compactification of $\mathbf{S}^{n} \backslash K$.

Let us consider some basic examples. If $K$ consists of only a single point, then $\mathbf{R}^{n} / K$ is automatically the same as $\mathbf{R}^{n}$ itself, and there is nothing to do. If $K$ is a finite set with more than one element, then it is easy to see that $\mathbf{R}^{n} / K$ is not a manifold. If we let $q$ denote the point in $\mathbf{R}^{n} / K$ which corresponds to $K$, then $\left(\mathbf{R}^{n} / K\right) \backslash\{q\}$ does not enjoy the local connectedness property that it should if $\mathbf{R}^{n} / K$ were a manifold at $q$, as in (3.3) in Section 3. More precisely, this local connectedness property for the complement of $\{q\}$ would be necessary only when $n \geq 2$. When $n=1$, one does not have to have this local connectedness condition, but then $\left(\mathbf{R}^{n} / K\right) \backslash\{q\}$ would have too many local components near $q$ for $\mathbf{R}^{n} / K$ to be a manifold at $q$. (That is, there would be more than 2 such local components.)

Now suppose that $K$ is a straight line segment in $\mathbf{R}^{n}$. In this event, $\mathbf{R}^{n} \backslash K$ is homeomorphic to $\mathbf{R}^{n}$ again. This is not hard to check. This would also work if $K$ were a standard rectangular cell of higher dimension in $\mathbf{R}^{n}$.

More generally, this works if $K$ is a tame cell in $\mathbf{R}^{n}$, meaning the image of a standard rectangular cell under a homeomorphism of $\mathbf{R}^{n}$ onto itself. This follows automatically from the case of standard rectangular cells.

However, if one merely assumes that $K$ is homeomorphic to a standard rectangular cell, then it is not necessarily true that $\mathbf{R}^{n} / K$ is a manifold! This is another aspect of wild embeddings, from Section 1. We shall say more about this as this section goes on. A concrete example is given by taking $K$ to be a copy of the Fox-Artin wild arc in $\mathbf{R}^{3}$. (Compare with $[$ Fer $]$.)

Note that we are not saying that $\mathbf{R}^{n} / K$ is always not a manifold when $K$ is wildly embedded. The converse is true, that $K$ must be wildly embedded when $\mathbf{R}^{n} / K$ is not a manifold (and $K$ is a topological 
cell). This is just a rephrasal of the remark above, that $\mathbf{R}^{n} / K$ is a manifold when $K$ is a tamely embedded cell.

Here is a slightly more foolish example, which one might view as a generalization of the earlier comments about the case where $K$ is a finite set with more than a single point. Imagine now that $K$ is a copy of the $j$-dimensional sphere $\mathbf{S}^{j}, 1 \leq j \leq n-1$. For this let us use a standard, smooth, round sphere; it is not a matter of wildness that we want to consider.

In this case $\mathbf{R}^{n} / K$ is never a topological manifold. If $j=n-1$, then $\mathbf{R}^{n} / K$ is homeomorphic to the union of $\mathbf{R}^{n}$ and an $n$-sphere, with the two meeting at a single point. This point is the one that corresponds to $K$ in $\mathbf{R}^{n} / K$. Let us denote this point by $q$ again, as above. In this case $\left(\mathbf{R}^{n} / K\right) \backslash\{q\}$ does not have the right local-connectedness property at $q$ in order for $\mathbf{R}^{n} / K$ to be a manifold, as in (3.3) in Section 3 .

If $j=n-2$, then one runs into trouble with local simple-connectivity of $\left(\mathbf{R}^{n} / K\right) \backslash\{q\}$ at $q$, as in (3.4) in Section 3. For this one might think about the special case where $n=3$, so that $K$ is a standard circle in $\mathbf{R}^{3}$. It is easy to take small loops in $\mathbf{R}^{3} \backslash K$, lying close to $K$, which are nonetheless linked with $K$. These loops then project down into $\left(\mathbf{R}^{3} / K\right) \backslash\{q\}$, where they can be as close to the point $q$ as one likes, but they are never contractable in $\left(\mathbf{R}^{3} / K\right) \backslash\{q\}$ at all, let alone in small neighborhoods of $q$ (as in (3.4) in Section 3). This is the same as saying that these loops are not contractable inside of $\mathbf{R}^{n} \backslash K$, which is equivalent to $\left(\mathbf{R}^{n} / K\right) \backslash\{q\}$.

When $j<n-2$, then one has similar obstructions to $\mathbf{R}^{n} / K$ being a manifold, but in terms of the failure of higher-dimensional forms of local connectedness of $\left(\mathbf{R}^{n} / K\right) \backslash\{q\}$ (using homology or homotopy). This is analogous to the cases already described, when $j=n-1$ or $n-2$. We shall say more about this soon, but for the moment let us go on to some other matters.

For this example, where $K$ is taken to be a standard $j$-dimensional sphere, note that $\mathbf{R}^{n} / K$ itself is locally contractable at $q$. This is as opposed to connectedness properties of $\left(\mathbf{R}^{n} / K\right) \backslash\{q\}$, and it is analogous to what happens in the case of finite polyhedra. Specifically, for finite polyhedra one always has local contractability, but the behavior near a given point of punctured neighborhoods around that point is another matter. The latter is connected to the behavior of the codimension-1 link of the polyhedron around the given point, as in Section 3.

In the present case, where we have $\mathbf{R}^{n} / K$ with $K$ a standard round $j$-dimensional sphere, one can see the local contractability of $\mathbf{R}^{n} / K$ at 
the point $q$ (corresponding to $K$ ) as follows. In $\mathbf{R}^{n}$, one can take a tubular neighborhood of $K$, which is homeomorphic to the Cartesian product of the $j$-sphere $K$ and an $(n-j)$-dimensional ball. This neighborhood can be contracted onto $K$ in a simple way, and this leads to the local contractability of $\mathbf{R}^{n} / K$ at $q$.

Now let us consider the case of the Whitehead continuum, from Section 2. We should not really say the Whitehead continuum here, as there is some flexibility in the construction, which can lead to the resulting set $W$ not being pinned down completely. This ambiguity will not really cause trouble for us here, and we can work with any compact set $W$ in $\mathbf{R}^{3}$ which is obtained as in the procedure described in Section 2.

The set $W$ has the feature of being cell-like, as in the following definition.

Definition 4.2 (Cell-like sets). A compact set $K$ in $\mathbf{R}^{n}$ is said to be cell-like if $K$ can be contracted to a point inside of any neighborhood $U$ of itself in $\mathbf{R}^{n}$.

Compare with [Dave2], especially p. 120. That the Whitehead continuum $W$ is cell-like is not hard to see from the construction of $W$, as the intersection of a decreasing sequence of solid tori with certain properties. Specifically, for this the key point is that the $\ell$ th solid torus can be contracted to a point inside the previous one.

If $K$ is a topological cell, then $K$ is contractable to a point inside of itself, without using the extra bit of room provided by a small neighborhood of itself. This is also independent of the way that $K$ might be embedded into some $\mathbf{R}^{n}$, i.e., wildly or tamely.

For $W$, it is not true that $\mathbf{R}^{3} / W$ is a topological manifold. If we let $q$ denote the point in $\mathbf{R}^{3} / W$ corresponding to $W$, then $\left(\mathbf{R}^{3} / W\right) \backslash\{q\}$ is not locally simply-connected at $q$ (in the sense of the condition in Section 3, around (3.4)). In concrete terms, this means that there are loops in $\mathbf{R}^{3} \backslash W$ (which is the same as $\left(\mathbf{R}^{3} / W\right) \backslash\{q\}$ ) which lie as close to $W$ as one likes (in their entirety), but which cannot be contracted to a point in $\mathbf{R}^{3} \backslash W$ while remaining reasonably close to $W$.

These loops can be described concretely, as meridians in the solid tori whose intersection gives $W$. The loop from the solid torus $T_{j}$ can be filled with a disk inside $T_{j}$, but not without crossing the smaller torus $T_{j+1}$, or any of its successors. This comes back to the way that each $T_{\ell+1}$ is "clasped" inside of $T_{\ell}$. See [Dave2], [Kir] for more information (including Proposition 9 on p. 76 of [Dave2]).

In any event, the failure of the local simple-connectivity of $\left(\mathbf{R}^{3} / W\right) \backslash\{q\}$ at $q$ is equivalent to $\mathbf{S}^{3} \backslash W$ not being simply-connected at infinity, as in 
Section 2. This also follows the discussion in Section 3, and the comment just after (4.1).

This case is quite different from the one of embedding round spheres in $\mathbf{R}^{n}$, as discussed before. More precisely, let us compare the situation with $W$ and the example before where $K$ is a standard circle inside of $\mathbf{R}^{3}$. For the latter, there are loops in $\mathbf{R}^{3} \backslash K$ which lie as close to $K$ as one wants, and which are not contractable to a point in $\mathbf{R}^{3} \backslash K$ at all, let alone in a neighborhood of $K$. For $W$, one has that $\mathbf{S}^{3} \backslash W$ is contractable (as mentioned in Section 2), and this implies that $\mathbf{R}^{3} \backslash W$ is simply-connected. (This is a straightforward exercise.) Thus these loops near $W$ can be contracted to a point in $\mathbf{R}^{3} \backslash W$, if one allows oneself to go away from $W$ for the contraction.

Here is another aspect of this. Although one has these loops in $\mathbf{R}^{3} \backslash W$ which lie near $W$ but cannot be contracted to a point in $\mathbf{R}^{3} \backslash W$ while staying near $W$, these loops can be made homologically trivial in $\mathbf{R}^{3} \backslash W$ while staying near $W$. That is, one can fill the loops with surfaces inside $\mathbf{R}^{3} \backslash W$ while staying close to $W$, if one allows the surfaces to have handles (rather than simply being a disk, as in the case of homotopic triviality). This is something that one can easily see from the pictures (as in [Dave2], [Kir]). The basic idea is that one can fill the loops with disks, where the disks stay close to $W$, but also pass through $W$ (and so are not in $\left.\mathbf{R}^{3} \backslash W\right)$. However, one can avoid the intersection with $W$ by cutting out a couple of small holes in the disk, and attaching a handle to them which goes along the boundary of the solid torus in the next generation of the construction. Then $W$ will stay inside this next solid torus, throughout the rest of the construction, and this surface gives a way of filling the loop without intersecting $W$ (or being forced to go far away from it).

This kind of filling by surfaces does not work in the case where we take $K$ to be a standard circle in $\mathbf{R}^{3}$. In this situation, we have loops in $\mathbf{R}^{3} \backslash K$ which lie close to $K$, and which are linked homologically with the circle $K$. In other words, the linking number of the loop with $K$ is nonzero, and this linking number is a homological invariant which would vanish if the loop could be filled with a surface without intersecting $K$. (For more about "linking numbers", see $[$ BotT], [Bre1], [Fla], [Spa].)

Here is another feature of $W$, which distinguishes it from ordinary circles in $\mathbf{R}^{3}$ (or spheres in $\mathbf{R}^{n}$ more generally). Let us think of $W$ now as lying in $\mathbf{R}^{4}$ rather than $\mathbf{R}^{3}$, through the inclusion of $\mathbf{R}^{3}$ in $\mathbf{R}^{4}$ by taking the fourth coordinate to be 0 .

For $\mathbf{R}^{4}$, we have that $\mathbf{R}^{4} / W$ is a topological manifold (homeomorphic to $\left.\mathbf{R}^{4}\right)$. The basic point behind this is the following. In the realization 
of $W$ as the intersection of a decreasing sequence of solid tori in $\mathbf{R}^{3}$, the th solid torus was always "clasped" in the previous one (as in Section 2, and [Dave2], [Kir]). In $\mathbf{R}^{4}$, the extra dimension provides a lot of extra room, in such a way that this "clasping" is not really present any more. If $T^{\prime}$ is a solid torus which is embedded and clasped inside of another solid torus $T$ in $\mathbf{R}^{3}$, one can "unclasp" $T^{\prime}$ in $\mathbf{R}^{4}$ by lifting one end up, bringing it around the hole in $T$, and leaving the other end alone. This is a standard observation, and it is analogous to the way that knots in $\mathbf{R}^{3}$ become unknotted in $\mathbf{R}^{4}$.

In other words, this procedure gives a way to make a deformation of $\mathbf{R}^{4}$, in which the solid torus $T^{\prime}$ is mapped to a set of small diameter, while not moving points some distance away at all. By contrast, back in $\mathbf{R}^{3}$, it is not possible to make an isotopy which shrinks $T^{\prime}$ to a set of small diameter, while leaving the points in the complement of the larger solid torus fixed. This is exactly because of the way that $T^{\prime}$ is "clasped" in $T$, so that it cannot be "unclasped" by an isotopy in $T$. When one has the extra dimension in $\mathbf{R}^{4}$, one can "undo" the clasping, by lifting one end up and moving it around, as indicated above.

Once one has this kind of "shrinking" in $\mathbf{R}^{4}$, one can use this to show that $\mathbf{R}^{4} / W$ is homeomorphic to $\mathbf{R}^{4}$. One can do this directly, using shrinking homeomorphisms like this, and combinations of them, to make a mapping from $\mathbf{R}^{4}$ to itself which shrinks $W$ to a point while remaining injective (and continuous) everywhere else. One puts homeomorphisms like this on top of each other, and deeper and deeper in the construction of $W$, until $W$ itself is shrunk all the way to a point. The various solid tori $T_{j}$ in the construction, of which $W$ is the intersection, are made smaller and smaller in this process. The trick is to do this without shrinking everything, so that the mapping that results remains a homeomorphism on the complement of $W$.

This idea of shrinking can be given a general form, and is discussed in detail in [Dave2]. See also [Edw2], [Kir].

By contrast, let us consider the case of a circle $K$ in $\mathbf{R}^{3}$. If one views $K$ as a subset of $\mathbf{R}^{4}$ in the same way, then $\mathbf{R}^{4} / K$ is still not a topological manifold. This follows from our earlier discussion about circles and spheres of higher dimensions inside of $\mathbf{R}^{n}$ in general. One also does not get a manifold by replacing $\mathbf{R}^{4}$ with $\mathbf{R}^{m}$ for larger $m$ 's.

Notice, however, that there is a kind of "improvement" that occurs in adding dimensions in this way. If $K$ is a circle in $\mathbf{R}^{3}$, and if $q$ denotes the point in $\mathbf{R}^{3} / K$ which corresponds to $K$, then $\left(\mathbf{R}^{3} / K\right) \backslash\{q\}$ is not locally simply-connected at $q$. For that matter, $\left(\mathbf{R}^{3} / K\right) \backslash\{q\} \cong \mathbf{R}^{3} \backslash K$ is not simply-connected at all. When one considers $K$ as a subset of 
$\mathbf{R}^{4}$, and asks analogous questions for $\mathbf{R}^{4} / K$ (or $\mathbf{R}^{4} \backslash K$ ), then there is no longer any trouble with simple-connectivity. The basic underlying problem continues, though, in the form of 2-dimensional connectivity. This is not hard to see.

Similarly, if one views $K$ as a subset of $\mathbf{R}^{n}$ for larger $n$, then the trouble with connectivity in lower dimensions goes away, but $(n-2)$-dimensional connectivity still does not work.

With the Whitehead continuum we are more fortunate. The problem with local simple-connectivity goes away when we proceed from $\mathbf{R}^{3}$ to $\mathbf{R}^{4}$, and difficulties with higher-dimensional connectivity do not then arise in their place. One should not be too surprised about this, since the Whitehead continuum is cell-like, while circles or spheres of higher dimension are not at all cell-like. In other words, with circles or spheres (and their complements in $\mathbf{R}^{n}$ ), there is some clear and simple nontrivial topology around, while the Whitehead continuum is much closer to something like a standard cell, which causes less trouble.

\subsection{Cellularity, and the cellularity criterion.}

Now let us look at some general notions and results, concerning the possibility that $\mathbf{R}^{n} / K$ be a topological manifold (and, in fact, homeomorphic to $\mathbf{R}^{n}$ ).

Definition 4.3 (Cellularity). A compact set $K$ in $\mathbf{R}^{n}$ (or, more generally, an $n$-dimensional topological manifold) is said to be cellular if it can be realized as the intersection of a countable family of sets $B_{i}$, where each $B_{i}$ is a topological $n$-cell (or, equivalently, homeomorphic to the closed unit ball in $\mathbf{R}^{n}$ ), and if each $B_{i+1}$ is contained in the interior of the preceding $B_{i}$.

Compare with [Dave2], especially p. 35, [Edw2], and p. 44 of [Rus1]. Alternatively, a compact set $K$ is cellular if and only if any neighborhood of $K$ contains an open set which contains $K$ and is homeomorphic to the standard $n$-dimensional ball.

Theorem 4.4. Let $K$ be a compact subset of $\mathbf{R}^{n}$. Then $\mathbf{R}^{n} / K$ is a topological manifold if and only if $K$ is cellular in $\mathbf{R}^{n}$. In this case, $\mathbf{R}^{n} / K$ is homeomorphic to $\mathbf{R}^{n}$.

See Exercise 7 on p. 41 of [Dave2] for the first assertion, and Proposition 2 on p. 36 of [Dave2] for the second one. (Concerning the latter, see Section 5 in [Dave2] too. Note that some of the notation in Exercise 7 on p. 41 in [Dave2] is explained in the statement of Proposition 2 on 
p. 36 of [Dave2].) See also [Edw2], especially the theorem on p. 114, and p. $44 \mathrm{ff}$ of [Rus1].

For the record, let us mention the following.

Proposition 4.5. Let $K$ be a compact subset of $\mathbf{R}^{n}$. If $K$ is cellular, then $K$ is cell-like. Conversely, if $n$ is equal to 1 or 2 , then $K$ is cellular if it is cell-like.

The fact that cellularity implies cell-likeness follows easily from the definitions. When $n=1$, the converse is very simple, since connectedness implies that a set is an interval, and hence cellular. In $\mathbf{R}^{2}$, the argument uses special features of plane topology. See Corollary $4 \mathrm{C}$ on p. 122 of [Dave2].

In higher dimensions, cell-like sets need not be cellular. Examples are given by Whitehead continua, and some wild embeddings of cells. However, there is an exact characterization of cellular sets among cell-like sets, which is the following. Basically, the point is to include the same kind of localized simple-connectivity of $\mathbf{R}^{n} \backslash K$ around $K$ as discussed before.

Theorem 4.6. Let $K$ be a compact set in $\mathbf{R}^{n}$, with $n \geq 3$. Then $K$ is cellular inside of $\mathbf{R}^{n}$ if and only if (a) it is cell-like, and (b) for every open neighborhood $U$ of $K$ in $\mathbf{R}^{n}$ there is another open neighborhood $V$ of $K$ so that every continuous mapping from $\mathbf{S}^{1}$ into $V \backslash K$ can be contracted to a point inside of $U \backslash K$.

This characterization of cellularity is stated in Theorem 5 on p. 145 of [Dave2]. This uses also the definition of the cellularity criterion given on p. 143 of [Dave2]. When $n \geq 4$, this result works for subsets of general $n$-dimensional topological manifolds, and not just $\mathbf{R}^{n}$. When $n=3$, there is trouble with the general case of manifolds, related to the 3 -dimensional Poincaré conjecture being unsettled; if the cellularity criterion holds for general manifolds, then the 3-dimensional Poincaré conjecture would follow, as discussed on p. 145 of [Dave2]. See Theorem 1.11 on p. 373 of $[$ Fre $]$ concerning the 4 -dimensional case, and $[\mathbf{M c M}]$ and Section 4.8 of [Rus1] for dimensions 5 and higher.

Corollary 4.7. Let $K$ be a compact subset of $\mathbf{R}^{n}, n \geq 3$. If $K$ is celllike in $\mathbf{R}^{n}$, then $K \times\{0\}$ is cellular in $\mathbf{R}^{n+1}$.

See Corollary $5 \mathrm{~A}$ on p. 145 of [Dave2]. The main point behind the derivation of Corollary 4.7 from Theorem 4.6 is that by passing to a Euclidean space of one higher dimension, potential trouble with local simple-connectedness of the complement of $K$ goes away. This fits with basic examples, and the Whitehead continuum in particular. 
Let us note the following simple converse to Corollary 4.7.

Lemma 4.8. Suppose that $K$ is a compact subset of $\mathbf{R}^{n}$. If $K \times\{0\}$ is cellular in $\mathbf{R}^{n+1}$, then $K$ is cell-like in $\mathbf{R}^{n}$.

Indeed, if $K \times\{0\}$ is cellular in $\mathbf{R}^{n+1}$, then it is also cell-like in $\mathbf{R}^{n+1}$, as in Proposition 4.5. It is easy to check that cell-likeness for $K \times\{0\}$ in $\mathbf{R}^{n+1}$ implies cell-likeness for $K$ inside $\mathbf{R}^{n}$, just by the definitions. (Thus cell-likeness, unlike cellularity, is not made more feasible by the extra room of extra dimensions.) This implies Lemma 4.8.

For concrete examples of cell-like sets, often the cellularity in higherdimensional spaces, as in Corollary 4.7, can be seen in fairly direct and simple terms. The room from the extra dimensions makes it easy to move pieces of the set apart, without the claspings, knottings, etc., which occurred originally. Some aspects of this came up earlier, concerning Whitehead continua.

Note that the localized simple-connectivity conditions that are used here are a bit different from those employed in the context of taming theorems, as in Section 1. To make this precise, let $K$ be a compact subset of some $\mathbf{R}^{n}$. The conditions that come up in the present section involve the behavior of $\mathbf{R}^{n} \backslash K$, localized around $K$ (the whole of $K$ ). That is, one looks at the behavior of $\mathbf{R}^{n} \backslash K$ within arbitrarily-small neighborhoods of $K$ in $\mathbf{R}^{n}$. In the context of Section 1, one would look at the behavior of $\mathbf{R}^{n} \backslash K$ near individual points in $K$.

To put it another way, here one seeks to contract loops in $\mathbf{R}^{n} \backslash K$ that are close to $K$ to points, while staying close to $K$. In the context of Section 1, one looks at small loops in $\mathbf{R}^{n} \backslash K$ near $K$, and tries to contract them to points in the complement of $K$ while staying in small balls, and not just staying near $K$.

\section{Manifold factors}

Let $W$ be a Whitehead continuum, constructed through a decreasing sequence of solid tori in $\mathbf{R}^{3}$, as in Section 2 .

Theorem 5.1. If $\mathbf{R}^{3} / W$ is defined as in Section 4 , then $\left(\mathbf{R}^{3} / W\right) \times \mathbf{R}$ is homeomorphic to $\mathbf{R}^{4}$.

In particular, $\left(\mathbf{R}^{3} / W\right) \times \mathbf{R}$ is a topological manifold, even though $\mathbf{R}^{3} / W$ itself is not. Thus $\mathbf{R}^{3} / W$ is a manifold factor.

The fact that $\left(\mathbf{R}^{3} / W\right) \times \mathbf{R}$ is homeomorphic to $\mathbf{R}^{4}$ is given as Corollary $3 \mathrm{~B}$ on p. 84 of [Dave2]. See also [AndR], [Kir].

Note that the existence of a homeomorphism from $\left(\mathbf{R}^{3} / W\right) \times \mathbf{R}$ onto $\mathbf{R}^{4}$ is not the same as the observation mentioned in Section 4, that 
$\mathbf{R}^{4} /(W \times\{0\})$ is homeomorphic to $\mathbf{R}^{4}$. In considering $\left(\mathbf{R}^{3} / W\right) \times \mathbf{R}$, one is in effect taking $\mathbf{R}^{4}$, and then shrinking each copy $W \times\{u\}$ of $W$ to a point, where $u$ runs through all real numbers. For $\mathbf{R}^{4} /(W \times\{0\})$, one shrinks only a single copy of $W$ to a point.

Although the construction is more complicated for $\left(\mathbf{R}^{3} / W\right) \times \mathbf{R}$ than for $\mathbf{R}^{4} /(W \times\{0\})$, there are some common aspects. As before, one of the main points is that the solid tori in $\mathbf{R}^{3}$ which are "clasped" (inside of other solid tori) become unclasped in $\mathbf{R}^{4}$. With the extra dimension in $\mathbf{R}^{4}$, one can pick up one end of one of these tori, bring it around, and then lay it down again, so that the clasping is undone. For the present situation with $\left(\mathbf{R}^{3} / W\right) \times \mathbf{R}$, one performs this kind of action for all of the copies $W \times\{u\}$ of $W$ at once, $u \in \mathbf{R}$, rather than just a single copy. (Compare also with Section 6, and the general notion of decomposition spaces mentioned there.)

In Section 2, it was mentioned that $\mathbf{S}^{3} \backslash W$ is a contractable open set which is not homeomorphic to a 3-ball (because it is not simplyconnected at infinity), and that $\left(\mathbf{S}^{3} \backslash W\right) \times \mathbf{R}$ is homeomorphic to a 4-dimensional open ball. (See [Bin3], [Bin4], [Kir].) This result is similar in some ways to Theorem 5.1, but the conclusions are not quite the same either.

In this vein, let us make the following observation. As usual, denote by $q$ the (singular) point in $\mathbf{R}^{3} / W$ that corresponds to $W$. Let us write $L$ for the subset of $\left(\mathbf{R}^{3} / W\right) \times \mathbf{R}$ given by $\{q\} \times \mathbf{R}$. Thus $L$ is homeomorphic to a line.

Using a homeomorphism from $\left(\mathbf{R}^{3} / W\right) \times \mathbf{R}$ to $\mathbf{R}^{4}$, one gets an embedding of $L$ into $\mathbf{R}^{4}$. It is not hard to see that any such embedding of $L$ into $\mathbf{R}^{4}$ has to be wild. Just as $\mathbf{R}^{3} \backslash W$ is not locally simply-connected near $W$, if $\mathcal{L}$ denotes the image of $L$ in $\mathbf{R}^{4}$ by an embedding as above, then $\mathbf{R}^{4} \backslash \mathcal{L}$ is not locally simply-connected near $\mathcal{L}$. (Note that $\mathbf{R}^{4} \backslash \mathcal{L}$ is homeomorphic to $\left(\mathbf{R}^{3} \backslash W\right) \times \mathbf{R}$, by construction.) This ensures that $\mathcal{L}$ is wild in $\mathbf{R}^{4}$, no matter what homeomorphism from $\left(\mathbf{R}^{3} / W\right) \times \mathbf{R}$ onto $\mathbf{R}^{4}$ one might use, since ordinary straight lines in $\mathbf{R}^{4}$ do not behave in this way.

One can also make local versions of this argument, to show that $\mathbf{L}$ is locally wild in the same manner.

If one were to want to pass from a homeomorphism from $\left(\mathbf{R}^{3} / W\right) \times \mathbf{R}$ onto $\mathbf{R}^{4}$ in Theorem 5.1 to a homeomorphism from $\left(\mathbf{S}^{3} \backslash W\right) \times \mathbf{R}$ onto $\mathbf{R}^{4}$, then in particular one could be lead to try to figure out something about what happens when one deletes $\mathcal{L}$ from $\mathbf{R}^{4}$. Conversely, if one wanted to go in the other direction, one might have to figure out something about 
how to put the topological line back in. These endeavors should be at least somewhat complicated, because of the wildness of $\mathcal{L}$ inside of $\mathbf{R}^{4}$.

The next fact helps to give an idea of how wild $\mathcal{L}$ can have to be.

Theorem 5.2. Let $U$ be an open set in some $\mathbf{R}^{n}$, and let $F$ be a closed set in $\mathbf{R}^{n}$. If the Hausdorff dimension of $F$ is less than $n-2$, then any open loop in $U \backslash F$ that can be contracted to a point in $U$ can also be contracted to a point in $U \backslash F$. In particular, $\mathbf{R}^{n} \backslash F$ is simply-connected.

See $[\mathbf{F a l}],[\mathbf{F e d}]$, [Mat] concerning the definition and basic properties of Hausdorff dimensions.

In other words, if $F \subseteq \mathbf{R}^{n}$ is closed and has Hausdorff dimension < $n-2$, then $F$ is practically invisible for considerations of fundamental groups, even local ones. From this one can check that $\mathcal{L}$ as above necessarily has Hausdorff dimension at least 2 , no matter the homeomorphism from $\left(\mathbf{R}^{3} / W\right) \times \mathbf{R}$ onto $\mathbf{R}^{4}$ which produced it. This is also true locally, i.e., each nontrivial arc of $\mathcal{L}$ has to have Hausdorff dimension at least 2 , and for the same reasons. This is somewhat remarkable, since $\mathcal{L}$ is homeomorphic to a line (and straight lines have Hausdorff dimension 1). (In general, Hausdorff dimension need not be preserved by homeomorphisms, though, and this is an instance of that.)

Theorem 5.2 is given (in a slightly different form) in [MarRV], in Lemma 3.3 on p. 9. See also [Geh2], [LuuV], [SieS], [Väi2] for related results. In particular, [SieS] uses Theorem 5.2 in a manner very similar to this, in the context of double-suspension spheres and homeomorphic parameterizations of them.

Note that instead of requiring that $F$ have Hausdorff dimension less than $n-2$ in Theorem 5.2, it is enough to ask that the $(n-2)$-dimensional Hausdorff measure of $F$ be zero.

By now, there are many examples known of spaces which are manifold factors (and not manifolds). The original discovery was in [Bin3], [Bin4], using a different space. This example, Bing's "dogbone" space, will come up again in Section 6 .

As mentioned near the bottom of p. 93 of [Dave2], 3 is the smallest dimension in which this type of phenomenon can occur (where a nonmanifold becomes a manifold after taking the Cartesian product with R), because of results about recognizing manifolds in dimensions 1 and 2. It does occur in all dimensions greater than or equal to 3 .

As a basic class of examples, if $K$ is a compact set in $\mathbf{R}^{n}$ which is a cell, then $\mathbf{R}^{n} / K$ may not be a manifold if $K$ is wild, but $\left(\mathbf{R}^{n} / K\right) \times \mathbf{R}$ is homeomorphic to $\mathbf{R}^{n+1}$. See [AndC], [Bry1], [Bry2], [Dave2], [Rus1]. 
More generally, if $K$ is any compact set in $\mathbf{R}^{n}$ which is cell-like, then $\left(\mathbf{R}^{n} / K\right) \times \mathbf{R}$ is homeomorphic to $\mathbf{R}^{n+1}$. When $n=1$ or $2, \mathbf{R}^{n} / K$ is itself homeomorphic to $\mathbf{R}^{n}$. (Compare with Proposition 4.5 and Theorem 4.4 in Subsection 4.1.) See Proposition 2 on p. 206 of [Dave2] for $n=3$, and Theorem 9 on p. 196 or Theorem 13 on p. 200 of [Dave2] for $n \geq 4$. (For Theorem 9 on p. 196 of [Dave2], note that the definition of a " $k$-dimensional decomposition" of a manifold is given near the top of p. 152 of [Dave2].)

Another class of examples (which is in fact closely related to the previous ones) comes from the celebrated double-suspension theorems of Edwards and Cannon [Can1], [Can2], [Can3], [Dave2], [Edw2]. From these one has the remarkable fact that there are finite polyhedra $P$ which are not topological manifolds, but for which $P \times \mathbf{R}$ is a manifold.

There are compact sets $K$ in $\mathbf{R}^{n}$ such that $K$ is not cell-like, and not cellular in particular, but $\left(\mathbf{R}^{n} / K\right) \times \mathbf{R}$ is homeomorphic to $\mathbf{R}^{n+1}$. This happens for every $n \geq 4$. See Corollary $3 \mathrm{E}$ on p. 185 of [Dave2]. (The proof uses the double-suspension theorems.)

There are also non-manifold spaces which become manifolds after taking the product with other non-manifold spaces. See Section 29 of [Dave2], beginning on p. 223.

\section{Decomposition spaces, 2}

The construction of the quotient $\mathbf{R}^{n} / K$, given a compact subset $K$ of $\mathbf{R}^{n}$, as in Section 4, is an example of a decomposition space. More generally, one can allow many subsets of $\mathbf{R}^{n}$ (or some other space) to be contracted to points at the same time, rather than just a single set.

In general, a decomposition of $\mathbf{R}^{n}$, or some other space, means a partition of it, i.e., a collection of subsets which are pairwise disjoint, and whose union is the whole space. One can then form the corresponding quotient space, first as a set - by collapsing the sets in the partition to individual points - and then as a topological space. The topology on the quotient is the richest one (the one with the most open sets) so that the canonical mapping from the space to the quotient is continuous.

All of this makes sense in general, but in order to have some good properties (like the Hausdorff condition for the quotient space), some assumptions about the decomposition should be made. As a start, typical assumptions would be that the individual sets that make up the decomposition be closed, and that the decomposition satisfy a certain upper semi-continuity property. See [Dave2] for details, including the 
definition on p. 13 of [Dave2]. For the present discussion, we shall always assume that some conditions like these hold, even if we do not say so explicitly.

If $G$ is a decomposition of $\mathbf{R}^{n}$, then one writes $\mathbf{R}^{n} / G$ for the corresponding quotient space.

Given a set $K$ in $\mathbf{R}^{n}$, one can always consider the decomposition of $\mathbf{R}^{n}$ consisting of $K$ and sets with only single elements, with the latter running through all points in $\mathbf{R}^{n} \backslash K$. This decomposition is sometimes denoted $G_{K}$, and the quotient $\mathbf{R}^{n} / G_{K}$ in the general sense of decompositions is the same as the space $\mathbf{R}^{n} / K$ from Section 4 .

As another basic situation, product spaces of the form $\left(\mathbf{R}^{n} / K\right) \times \mathbf{R}$ can be viewed as decomposition spaces. Specifically, one gets a decomposition of $\mathbf{R}^{n+1} \cong \mathbf{R}^{n} \times \mathbf{R}$ using sets of the form $K \times\{u\}$ in $\mathbf{R}^{n+1}$ for each $u \in \mathbf{R}$, together with single-element sets for all of the points in $\mathbf{R}^{n+1} \backslash(K \times \mathbf{R})$. The resulting decomposition space is equivalent topologically to $\left(\mathbf{R}^{n} / K\right) \times \mathbf{R}$.

An important general point is that wild or interesting embeddings can often occur in simple or useful ways through decompositions. For instance, in the decomposition space described in the preceding paragraph, one has a particular "line", corresponding to the copies of $K$. See [Can1], [Dave1], [Dave2] for more information, including p. 451 of [Can1], the last paragraph in Section 2 on p. 380 of [Dave1], and the remarks near the top of p. 37 in [Dave2].

The following theorem of R. L. Moore [Moo] is an early result about when decomposition spaces are manifolds, and homeomorphic to the original space.

Theorem 6.1. Let $X$ be a compact Hausdorff topological space. Suppose that $f$ is a continuous mapping from the 2-sphere $\mathbf{S}^{2}$ to $X$, and that for each $x \in X, f^{-1}(x)$ is nonempty and connected, and $\mathbf{S}^{2} \backslash f^{-1}(x)$ is nonempty and connected. Then $X$ is homeomorphic to $\mathbf{S}^{2}$.

In this theorem, the mapping $f$ itself may not be a homeomorphism. As in Section 4, $f$ might have the effect of collapsing some line segments down to single points, for instance. It is true that $f$ can always be approximated by homeomorphisms, however. See [Dave2] for more information and references.

Similar results hold in dimension 1. For this it is enough to assume that the inverse images of points under the mapping be connected (and nonempty proper subsets of the domain), without imposing conditions on their complements. In this situation, the inverse images of points will 
simply be arcs. Compare with [Dave2], including the remarks near the bottom of p. 17 .

What might be reasonable analogues of Theorem 6.1 in higher dimensions? One should not keep the hypotheses literally as they are above, where the fibers are connected and have connected complements, because of counterexamples like the non-manifold spaces that one can get by contracting a circle to a point (as in Section 4).

However, in dimension 2, the property of a set in $\mathbf{S}^{2}$ or $\mathbf{R}^{2}$ being connected and having connected complement is quite strong. For a closed subset of $\mathbf{S}^{2}$ which is not empty nor all of $\mathbf{S}^{2}$, these conditions imply that the complement of the set is homeomorphic to a 2-dimensional disk, and that the set itself is cellular (Definition 4.3 in Section 4).

A decomposition $G$ of $\mathbf{R}^{n}$ is said to be cellular if each of the subsets of $\mathbf{R}^{n}$ of which it is composed is cellular. (Compare with [Dave2], in the statement of Corollary 2A on p. 36.) As an analogy with Moore's theorem, one might hope that a quotient of $\mathbf{R}^{n}$ (or $\mathbf{S}^{n}$, or other topological manifolds) by a cellular decomposition is a manifold, and homeomorphic to $\mathbf{R}^{n}$ again (or to the original manifold, whatever it might be).

This is true for decompositions which consist of a single cellular subset of the space, together with all the remaining points in the space as one-element sets. In other words, this statement is true in the context of quotients as in Section 4. See Theorem 4.4 in Section 4, and Proposition 2 on p. 36 of [Dave2].

For decompositions in general, it is not true that cellularity is sufficient to ensure that the quotient space is a manifold. This fails already for decompositions of $\mathbf{R}^{3}$. The first example of this was provided by Bing's "dogbone" construction in [Bin2]. See also [Dave2], especially Section 9, for this and other examples. For the statement that the decomposition space is not a manifold, and not just not homeomorphic to $\mathbf{R}^{3}$, see Theorem 13 on p. 498 of [Bin2]. A recent paper related to this is $[$ Arm $]$.

The dogbone space was also used in the initial discovery of manifold factors. See [Bin3], [Bin4].

Although quotients by cellular decompositions do not in general give manifolds, there are many nontrivial examples where this does occur, and results about when it should take place. A particularly nice and fundamental example is given by "Bing doubling". See [Bin1], [Dave2] (Example 1 in Section 9 of [Dave2]). This is a decomposition of $\mathbf{R}^{3}$ for which the corresponding quotient space is homeomorphic to $\mathbf{R}^{3}$. While the quotient space is standard, the decomposition has some interesting features, giving rise to some wild embeddings in particular. This 
decomposition has a symmetry to it, which leads to a homeomorphic involution on $\mathbf{S}^{3}$ which is highly nonstandard. The fixed-point set of this homeomorphism is a wildly-embedded 2-sphere in $\mathbf{S}^{3}$. This construction apparently gave the first examples of involutions on $\mathbf{R}^{3}$ which were not topologically equivalent to "standard" ones, made from rotations, reflections, and translations. See [Bin1] for more information, especially Section 4 of [Bin1].

See Section 9 of [Bin4] concerning a wild involution on $\mathbf{R}^{4}$, whose fixed point set is homeomorphic to the dogbone space. This uses the fact that the product of the dogbone space with the real line is homeomorphic to $\mathbf{R}^{4}$.

The results mentioned in Section 5 -about quotients $\mathbf{R}^{n} / K$ being homeomorphic to a Euclidean space after taking the Cartesian product with $\mathbf{R}$ - can be viewed as providing nontrivial examples of cellular decompositions of $\mathbf{R}^{n+1}$ for which the corresponding quotients are manifolds, and homeomorphic to $\mathbf{R}^{n+1}$.

\section{Geometric structures for decomposition spaces}

\subsection{A basic class of constructions.}

One feature of decomposition spaces is that they do not a priori come with a canonical or especially nice geometry, or anything like that. The topology is canonical, but this is somewhat different. Note that there are general results about existence of metrics which are compatible with the topology, as in Proposition 2 on p. 13 of [Dave2]. Once one has one such metric, there are many which define the same topology. This is true just as well for ordinary Euclidean spaces, or the spheres $\mathbf{S}^{n}$, even if there are also special metrics (like the Euclidean metric) that one might normally like or use.

In some cases (for decomposition spaces), there are some particularly nice or special geometries that one can consider. A number of basic examples - like the Whitehead continuum, Bing doubling [Bin1], and Bing's dogbone space [Bin2] — have a natural topological "selfsimilarity" to them, which can be converted into geometric self-similarity.

Let us be more precise. In these cases, the nondegenerate elements of the decomposition are generated by repeating a simple "rule". The "rule" can be described by a smooth domain $D$ in $\mathbf{R}^{n}$ together with some copies of $D$ embedded in the interior of $D$, in a pairwise-disjoint manner. To generate the nontrivial elements of the decomposition, one starts with $D$, and then passes to the copies of $D$ inside of itself. For each of these copies of $D$ inside of $D$, one can get a new collection of 
smaller copies of $D$, by applying the "rule" to the copies of $D$ with which we started. One then repeats this indefinitely.

Thus, if the original "rule" involves $m$ copies of $D$ inside of itself, then the $j$ th step of this process gives rise to $m^{j-1}$ copies of $D$, with the first step corresponding to $D$ alone.

The limiting sets which arise from this procedure are pairwise disjoint, and are used to define the decomposition. To do this carefully, one can think of the $j$ th step of the process as producing a compact set $C_{j}$, which is the union of the $m^{j-1}$ individual copies of $D$ indicated above. The construction gives $C_{j} \subseteq C_{j-1}$ for all $j \geq 2$. To pass to the limit, one can take the set $C=\bigcap_{j=1}^{\infty} C_{j}$, and then use the components of $C$ as subsets of $\mathbf{R}^{n}$ to be employed in the decomposition $G$ of $\mathbf{R}^{n}$. For each point $x \in \mathbf{R}^{n} \backslash C$, one also includes the one-point set $\{x\}$ in the decomposition $G$.

The Whitehead continuum (discussed in Section 2) is an example of this. There the "rule" is particularly simple, in that it is based on an embedding of a single solid torus $T_{1}$ inside a larger one $T$. At each stage of the process there is only one domain, and only one nondegenerate set being produced in the end (i.e., the Whitehead continuum). In particular, one can have $m=1$ in the general set-up described above, and with the result being nontrivial. For Bing doubling and Bing's dogbone space, one has $m>1$, i.e., there are more than one embedding being used at each step, and more than one copy of the basic domain. (For Bing doubling the basic domain is again a solid torus, while for the dogbone space it is a solid 2-handled torus. In Bing doubling one has $m=2$, as the name suggests. For the dogbone space, $m=4$.) In these cases the number of components grows exponentially in the process, and is infinite in the end (after taking the limit).

At any rate, there are a number of basic examples of decompositions generated in this manner, with individual copies of a domain being replaced systematically by some embedded subdomains, copies of itself, following repetitions of a single basic "rule". See [Dave2], especially Section 9 .

In typical situations, the basic "rule" involves nontrivial distortion of the standard Euclidean geometry at each step. As a first point along these lines, when one embeds a copy of some (bounded) domain $D$ into itself, then some change in the geometry (along the lines of shrinkage) is unavoidable, at least if the embedded copy is a proper subset of the original domain.

Given that there needs to be some shrinkage in the embedding, the next simplest possibility would be that the embeddings are made up out 
of something like dilations, translations, rotations, and reflections. In other words, except for a uniform scale factor, one might hope that the geometry does not have to change.

Normally this will not be the case. Some amount of bending or twisting, etc., will (in general) be involved, and needed, to accommodate the kind of topological behavior that is present. This includes linking, clasping, or things like that.

For the purpose of choosing a geometry that might fit with a given decomposition space, however, one can modify the usual Euclidean metric so that the embeddings involved in the basic "rule" do have the kind of behavior indicated above, i.e., a constant scale factor together with an isometry. The scale factors should be less that 1 , to reflect the shrinking that is supposed to take place for the decomposition spaces (even at a purely topological level).

It is not hard to see that one can make deformations of geometry like this. One can do this in a kind of direct and "intrinsic" way, defining metrics on $\mathbf{R}^{n}$ with suitable properties. One can also do this through embeddings of the decomposition spaces into higher-dimensional Euclidean spaces. In these higher-dimensional Euclidean spaces, the self-similarity that one wants, in typical situations, can be realized in terms of standard linear self-similarity, through dilations and translations.

More precisely, in these circumstances, the quotient of $\mathbf{R}^{n}$ by the decomposition can be realized topologically as an $n$-dimensional subset $X$ of some $\mathbf{R}^{N}$ (with $N=n+1$, for instance), in such a way that $X$ is a smooth submanifold away from the natural singularities, and $X$ is self-similar around these singularities.

To build such a set $X$, one can start with the complement of the original domain $D$ in $\mathbf{R}^{n}$. One would view $\mathbf{R}^{n} \backslash D$ as an $n$-dimensional submanifold of $\mathbf{R}^{N}$. In place of the iteration of the basic rule for the decomposition from before, one now stacks some "basic building blocks" in $\mathbf{R}^{N}$ on top of $\mathbf{R}^{n} \backslash D$ (along the boundary of $D$ ), and then on top of the other building blocks, over and over again.

These basic building blocks are given by $n$-dimensional smooth manifolds in $\mathbf{R}^{N}$ (with boundary). They are diffeomorphic to a single "model" in $\mathbf{R}^{n}$, which is the original domain $D$ in $\mathbf{R}^{n}$, minus the interiors of the $m$ copies of $D$ embedded inside $D$, as given by the basic "rule" that generates the decomposition. The building blocks are all diffeomorphic to each other, since they are all diffeomorphic to this same model, but they are also constructed in such a way as to be "similar" to each other. That is, they can all be given by translations and dilations of each other. 
This is a key difference between this construction and the original decomposition in $\mathbf{R}^{n}$.

Further, the building blocks are constructed in such a way that their ends are all similar to each other (i.e., even different ends on the same building block). Specifically, the building blocks are chosen so that when one goes to stack them on top of each other, their "ends" fit together properly, with smoothness across the interfaces.

These things are not difficult to arrange. Roughly speaking, one uses the extra dimensions in $\mathbf{R}^{N}$ to straighten the "ends" in this way, so that the different building blocks can be stacked properly. Typically, this would involve something like the following. One starts with the basic model in $\mathbf{R}^{n}$, given by $D$ minus the interiors of the $m$ embedded copies of $D$ in $D$. One then makes some translations of the $m$ embedded subdomains in $D$, up into the extra dimension or dimensions in $\mathbf{R}^{N}$. Up there, these subdomains can be moved or bent around, until they are similar to $D$ itself (i.e., being the same modulo translations and dilations). This can be done one at a time, and without changing anything near the boundary of the original domain $D$. In this manner, the original model region in $\mathbf{R}^{n}$ becomes realized as an $n$-dimensional compact smooth submanifold (with boundary) in $\mathbf{R}^{N}$, with the ends matching up properly under similarities.

To put it another way, the main "trade-off" here is that one gives up the "flatness" of the original model, as a region in $\mathbf{R}^{n}$, to get basic building blocks in $\mathbf{R}^{N}$ that are $n$-dimensional curved submanifolds whose ends are similar to each other. The curving of the interiors of these building blocks compensates for the straightening of their ends.

As above, one then stacks these building blocks on top of each other, one after another, to get a realization of the decomposition space by an $n$-dimensional subset $X$ of $\mathbf{R}^{N}$. (One also puts in some limiting points, at the ends of the towers of the building blocks that arise. In other words, this makes $X$ be a closed subset of $\mathbf{R}^{N}$. These extra points are the singularities of $X$.) This subset is smooth away from the singularities, and self-similar at the singularities, because of the corresponding properties of the basic building blocks.

By choosing the scale-factors associated to the ends of the basic building blocks to be less than 1 , the diameters of the ends tend to 0 (and in a good way) as one stacks the building blocks on top of each other many times. This corresponds to the fact that the sets in the decomposition are supposed to be shrunk to single points in the quotient space. This is also part of the story of the "limiting points" in the previous paragraph. The limiting points are exactly the ones associated (in the end) to the 
nondegenerate sets in the original decomposition in $\mathbf{R}^{n}$, which are being shrunk to single points.

The actual homeomorphic equivalence between the set $X$ in $\mathbf{R}^{N}$ produced through this method and the decomposition space $\mathbf{R}^{n} / G$ with which one starts is obtained using the diffeomorphic equivalence between the building blocks in $\mathbf{R}^{N}$ and the original model in $\mathbf{R}^{n}$ ( $D$ minus the interiors of the $m$ embedded copies of itself, as above). In rough terms, at the level of the topology, the same kind of construction is occurring in both places, $X$ and the decomposition space, and one can match them up, by matching up the individual building blocks. This is not hard to track.

Instead of stacking building blocks on top of each other infinitely many times, one can stop after finitely many steps of the construction (and add in suitable plugs to fill in the holes). This gives a set which is still smooth, and diffeomorphic to $\mathbf{R}^{n}$, and which approximates the non-smooth version that represents the decomposition space.

When one makes constructions like these - either finite approximations or infinite limits - the self-similarity helps to ensure that the spaces behave geometrically about as well as they could. See [Sem3] for more information, and some slightly different versions of these basic themes.

\subsection{Quotient spaces can be topologically standard, but geo- metrically tricky.}

We have seen before how decompositions of $\mathbf{R}^{n}$ might lead to $\mathbf{R}^{n}$ again topologically in the quotient, but do so in a manner that is still somehow nontrivial. For instance, the decomposition might arise from a nontrivial manifold factor, or lead to wild embeddings in the quotient which seem very simple (like a straight line) at the level of the decomposition. In these situations, one can still have highly nontrivial geometries from the procedures described in Subsection 7.1, even though the underlying space is topologically equivalent to $\mathbf{R}^{n}$.

As a special case, wild embeddings in the quotient can have nice metric properties in the kind of geometric realizations discussed here, while the same properties would not be possible in $\mathbf{R}^{n}$ with the standard Euclidean metric.

Here is a concrete instance of this. Let $W$ be a Whitehead continuum in $\mathbf{R}^{3}$, as in Section 2. Consider the corresponding quotient space $\mathbf{R}^{3} / W$, as in Section 4. One can realize $\mathbf{R}^{3} / W$ topologically as a subset of $\mathbf{R}^{4}$, where this subset is smooth away from the singular point, and has a simple self-similarity at the singular point, as in Subsection 7.1. Similarly, 
one can think of $\left(\mathbf{R}^{3} / W\right) \times \mathbf{R}$ as being given as a subset of $\mathbf{R}^{5}$, namely, as the product of the one in $\mathbf{R}^{4}$ with $\mathbf{R}$.

Let us write $q$ for the singular point in $\mathbf{R}^{3} / W$, i.e., the point in the quotient which corresponds to $W$, and set $L=\{q\} \times \mathbf{R}$. With respect to the embedding of $\left(\mathbf{R}^{3} / W\right) \times \mathbf{R}$ into $\mathbf{R}^{5}, L$ has Hausdorff dimension 1 , and bounded subsets of it have finite 1-dimensional Hausdorff measure. However, the image of $L$ inside of $\mathbf{R}^{4}$ under a homeomorphism from $\left(\mathbf{R}^{3} / W\right) \times \mathbf{R}$ onto $\mathbf{R}^{4}$ will be wild. As in Section 5 , the image of $L$ in $\mathbf{R}^{4}$ under such a homeomorphism has Hausdorff dimension at least 2, with respect to the usual Euclidean metric in $\mathbf{R}^{4}$. This uses Theorem 5.2.

This shows that the geometry that we have for $\left(\mathbf{R}^{3} / W\right) \times \mathbf{R}$ has to be substantially different from the usual Euclidean geometry on $\mathbf{R}^{4}$, even though the two spaces are topologically equivalent. Specifically, even though there are homeomorphisms from $\left(\mathbf{R}^{3} / W\right) \times \mathbf{R}$ onto $\mathbf{R}^{4}$, no such homeomorphism can be Lipschitz, or even Hölder continuous of order larger than 1/2. (Recall that a mapping is Lipschitz if for each pair of points in the domain, the distance between their images is bounded by a constant times the distance between the points themselves. A mapping is Hölder continuous of order $\alpha$ if the distance between the images of two points is bounded by a constant times the distance between the two original points raised to the power $\alpha$. For this condition, it is often natural to restrict one's attention to pairs of points which are no more than distance 1 apart, or to points in bounded regions.)

Although $\left(\mathbf{R}^{3} / W\right) \times \mathbf{R}$ - with the kind of geometry described aboveis quite different from $\mathbf{R}^{4}$ with the usual Euclidean metric, there is a strong and nice feature that it has, in common with $\mathbf{R}^{4}$. We shall call this property "uniform local coordinates".

Since $\left(\mathbf{R}^{3} / W\right) \times \mathbf{R}$ is homeomorphic to $\mathbf{R}^{4}$, it has homeomorphic local coordinates from $\mathbf{R}^{4}$ at every point. "Uniform local coordinates" asks for a stronger version of this, and is more quantitative. Specifically, around each metric ball $B$ in $\left(\mathbf{R}^{3} / W\right) \times \mathbf{R}$ (with respect to the kind of geometry that we have), there are homeomorphic local coordinates from a standard Euclidean ball $\beta$ of the same radius in $\mathbf{R}^{4}$, such that

the image of $\beta$ under the coordinate mapping

covers the given ball $B$ in $\left(\mathbf{R}^{3} / W\right) \times \mathbf{R}$,

and

the modulus of continuity of the coordinate mapping and its

inverse can be controlled, uniformly over all choices of metric

balls $B$ in $\left(\mathbf{R}^{3} / W\right) \times \mathbf{R}$, and in a scale-invariant manner. 
Here "modulus of continuity" means a function $\omega(r)$ so that when two points in the domain (of a given mapping) are at distance $\leq r$, their images are at distance $\leq \omega(r)$. Also, $r$ would range through positive numbers, and $\omega(r)$ would be nonnegative and satisfy

$$
\lim _{r \rightarrow 0} \omega(r)=0 .
$$

This last captures the continuity involved, and, in fact, gives uniform continuity.

For a mapping from a compact metric space to another metric space, continuity automatically implies uniform continuity, and that implies the existence of some modulus of continuity. This is not to say that one knows much about the modulus of continuity, a priori. (One can always choose it to be monotone, for instance, but one cannot in general say how fast it tends to 0 as $r \rightarrow 0$.)

Concrete examples of moduli of continuity would include $\omega(r)=C r$ for some constant $C$, which corresponds to a mapping being Lipschitz with constant $C$, or $\omega(r)=C r^{\alpha}, \alpha>0$, which corresponds to Hölder continuity of order $\alpha$. One can have much slower rates of vanishing, such as $\omega(r)=(\log \log \log (1 / r))^{-1}$.

In our case, with the property of "uniform local coordinates", we want to have a single modulus of continuity $\omega(r)$ which works simultaneously for all of the local coordinate mappings (and their inverses). Actually, we do not look at moduli of continuity for the mappings themselves, but renormalized versions of them. The renormalizations are given by dividing distances in the domain and range by the (common) radius of $B$ and $\beta$. In this way, $B$ and $\beta$ are viewed as though they have radius 1 , independently of what the radius was originally. This gives a kind of uniform basis for making comparisons between the behavior of the individual local coordinate mappings and their moduli of continuity.

Let us return now to the special case of $\left(\mathbf{R}^{3} / W\right) \times \mathbf{R}$, with the geometry as before. In this case one can get the condition of uniform local coordinates from the existence of topological coordinates (without uniform bounds), together with the self-similarity and smoothness properties of the set. Here is an outline of the argument. (A detailed version of this, for a modestly different situation, is given in [Sem3]. Specifically, see Theorem 6.3 on p. 241 in [Sem3]. Note that the property of uniform local coordinates is called "Condition $(* *)$ " in [Sem3], as in Definition 1.7 on p. 192 of [Sem3].)

Let $B$ be a metric ball in $\left(\mathbf{R}^{3} / W\right) \times \mathbf{R}$, for which one wants to find suitable coordinates. Assume first that $B$ does not get too close to the singular line $\{q\} \times \mathbf{R}$ in $\left(\mathbf{R}^{3} / W\right) \times \mathbf{R}$, and in fact that the radius of 
$B$ is reasonably small compared to the distance from $B$ to the singular line. In this case $\left(\mathbf{R}^{3} / W\right) \times \mathbf{R}$ is pretty smooth and flat in $B$, by construction (through the method of Subsection 7.1). This permits one to get local coordinates around $B$ quite easily, and with suitable uniform bounds for the moduli of continuity of the coordinate mappings and their inverses. The bounds that one gets are scale-invariant, because of the self-similarity in the geometric construction (from Subsection 7.1). In fact, one can have Lipschitz bounds in this case, as well as stronger forms of smoothness.

If the ball $B$ is reasonably close to the singular line $\{q\} \times \mathbf{R}$, then one can reduce to the case where it is actually centered on $\{q\} \times \mathbf{R}$. That is, one could replace $B$ with a ball which is centered on $\{q\} \times \mathbf{R}$, and which is not too much larger. (The radius of the new ball would be bounded by a constant times the radius of $B$.) This substitution does not cause trouble for the kind of bounds which are sought here.

Thus we suppose that $B$ is centered on the line $\{q\} \times \mathbf{R}$. We may as well assume that the center of $B$ is the point $(q, 0)$. This is because $\left(\mathbf{R}^{3} / W\right) \times \mathbf{R}$ and the geometry that we have on it are invariant under translations in the $\mathbf{R}$ direction, so that one can move the center to $(q, 0)$ without difficulty, if necessary.

Using the self-similarity of $\left(\mathbf{R}^{3} / W\right) \times \mathbf{R}$, one can reduce further to the case where the radius of $B$ is approximately 1 . For that matter, one can reduce to the case where it is equal to 1 , by simply increasing the radius by a bounded factor (which again does not cause problems for the uniform bounds that are being considered here). (To be honest, if one takes the geometry for $\mathbf{R}^{3} / W$ to be flat outside a compact set, as in Subsection 7.1, then this reduction is not fully covered by self-similarity. That is, one should handle large scales a bit differently. This can be done, and a similar point is discussed in [Sem3] in a slightly different situation (for examples based on "Bing doubling").)

Once one makes these reductions, one gets down to the case of the single ball $B$ in $\left(\mathbf{R}^{3} / W\right) \times \mathbf{R}$, centered at $(q, 0)$ and with radius 1 . For this single choice of scale and location, one can use the fact that $\left(\mathbf{R}^{3} / W\right) \times \mathbf{R}$ is homeomorphic to $\mathbf{R}^{4}$ to get suitable local coordinates.

For this single ball $B$, there is no issue of "uniformity" in the moduli of continuity for the coordinate mappings. One simply needs $a$ modulus of continuity.

A key point, however, is that when one works backwards in the reductions just made, to go to arbitrary balls in $\left(\mathbf{R}^{3} / W\right) \times \mathbf{R}$ which are relatively close to the singular line $\{q\} \times \mathbf{R}$, one does get local coordinates with uniform control on the (normalized) moduli of continuity of 
the coordinate mappings and their inverses. This is because of the way that the reductions cooperate with the scaling and the geometry.

At any rate, this completes the outline of the argument for showing that $\left(\mathbf{R}^{3} / W\right) \times \mathbf{R}$ has uniform local coordinates, in the sense described before, and with the kind of geometry for $\left(\mathbf{R}^{3} / W\right) \times \mathbf{R}$ as in Subsection 7.1.

It is easy to see that a metric space which is bilipschitz equivalent to some $\mathbf{R}^{n}$ has uniform local coordinates (relative to $\mathbf{R}^{n}$ rather than $\mathbf{R}^{4}$, as above). (Recall that two spaces are bilipschitz equivalent if there is a homeomorphism from one onto the other which is both Lipschitz and has Lipschitz inverse.) The required local coordinates can simply be obtained from restrictions of the global bilipschitz parameterization. The converse is not true in general, i.e., a space can have uniform local coordinates and not be bilipschitz equivalent to the corresponding $\mathbf{R}^{n}$. An example of this is given by $\left(\mathbf{R}^{3} / W\right) \times \mathbf{R}$ with the kind of geometry that we have been considering.

One can also get much simpler examples, by taking snowflake spaces. That is, one can take $\mathbf{R}^{n}$ equipped with the metric $|x-y|^{\alpha}$, where $|x-y|$ is the usual metric, and $\alpha$ is a positive real number strictly less than 1 . It is not hard to check that this has the property of uniform local coordinates. It is not bilipschitz equivalent to $\mathbf{R}^{n}$, because it has Hausdorff dimension $n / \alpha$ instead of Hausdorff dimension $n$. The previous example based on $\left(\mathbf{R}^{3} / W\right) \times \mathbf{R}$ behaves much better than this, though, with the correct Hausdorff dimension (namely, 4) in particular.

Notice that the uniform local coordinates property would imply a bilipschitz condition if the local coordinates all came from restrictions of a single global parameterization. This is because of the way that the scaling works. In general, the uniform local coordinates property allows the local coordinate mappings to change as one changes locations and scales, and this is why it allows for there to be no global bilipschitz parameterization. The case of $\left(\mathbf{R}^{3} / W\right) \times \mathbf{R}$ provides a good example of this.

Another way to think about the uniformity over all locations and scales in the uniform local coordinates property is that it is a condition which implies the existence of homeomorphic coordinates even after one "blows up" the space (in the Hausdorff or Gromov-Hausdorff senses) along any sequence of locations and scales in the space. With the uniform local coordinates property, the local coordinates could be "blown up" along with the space, with the uniform bounds for the moduli of continuity providing the equicontinuity needed to take limits of the coordinate mappings (after passing to suitable subsequences). 
Instead of looking at uniform local coordinates in connection with bilipschitz equivalence with $\mathbf{R}^{n}$, one can consider quasisymmetric equivalence. Roughly speaking, a quasisymmetric mapping between two metric spaces is one that approximately preserves relative distances, in the same way that bilipschitz mappings approximately preserve actual distances. In other words, if one has three points $x, y$, and $z$ in the domain of such a mapping, and if $x$ is much closer to $y$ than $z$ is, then this should also be true for their images under a quasisymmetric mapping, even if the actual distances between the points might be changed a lot. See [TukV] for more information about quasisymmetric mappings. Two metric spaces are quasisymmetrically equivalent if there is a quasisymmetric mapping from one onto the other. As with bilipschitz mappings, compositions and inverses of quasisymmetric mappings remain quasisymmetric.

If a metric space admits a quasisymmetric parameterization from $\mathbf{R}^{n}$, then it also satisfies the condition of uniform local coordinates. This is true for nearly the same reason as for bilipschitz mappings; given a quasisymmetric mapping from $\mathbf{R}^{n}$ onto the metric space, one can get suitable local coordinates for the space from restrictions of the global mapping to individual balls. There is a difference between this case and that of bilipschitz mappings, which is that one should allow some extra rescalings to compensate for the fact that distances are not approximately preserved. Specifically, a ball $B$ in the metric space may be covered in a nice way by the image under a quasisymmetric mapping of a ball $\beta$ in $\mathbf{R}^{n}$, but the radii of $B$ and $\beta$ need not match up. For a bilipschitz mapping, one would be able to choose $\beta$ so that it has radius which is comparable to that of $B$. In the quasisymmetric case, one may not have that, but if one adds an extra rescaling on $\mathbf{R}^{n}$ (depending on the choices of balls), then one can still get local coordinates with the kind of uniform control on the (normalized) moduli of continuity as in the uniform local coordinates condition.

If a metric space has uniform local coordinates with respect to $\mathbf{R}^{n}$, and if these coordinates come from restrictions and then rescalings (on $\mathbf{R}^{n}$ ) of a single global parameterization, as in the preceding paragraph, then that parameterization does have to be quasisymmetric. This is an easy consequence of the definitions, and it is analogous to what happens in the bilipschitz case.

If a metric space admits uniform local coordinates from some $\mathbf{R}^{n}$, it still may not be true that it admits a quasisymmetric parameterization. This is trickier than before, and in particular one does not get examples 
simply by using snowflake metrics $|x-y|^{\alpha}$ on $\mathbf{R}^{n}$. Indeed, the identity mapping on $\mathbf{R}^{n}$ is quasisymmetric as a mapping from $\mathbf{R}^{n}$ with the standard metric to $\mathbf{R}^{n}$ with the snowflake metric $|x-y|^{\alpha}, 0<\alpha<1$.

However, there are counterexamples, going back to results of Rickman and Väisälä. That is, these are spaces which have uniform local coordinates (and are even somewhat nicer than that), but which do not admit quasisymmetric parameterizations. Basically, these spaces are Cartesian products, where the individual factors can behave nicely in their own right, and where the combination mixes different types of geometry. A basic example (which was the original one) is to take a product of a snowflake with a straight line. Quasisymmetric mappings try to treat different directions in a uniform manner, and in the end this does not work for parameterizations of these examples. See Lemma 4 in [Tuk], and also [Väi3] and [AleV].

These examples occur already in dimension 2. They do not behave well in terms of measure, though. This is a basic part of the story; compare with [AleV], [Tuk], [Väi3].

As usual, dimension 1 is special. There are positive results starting from more primitive conditions, and good characterizations for the existence of quasisymmetric parameterizations, in fact. See Section 4 of [TukV].

In dimension 2 , there are positive results about having global quasisymmetric parameterizations for a given space, and with bounds, under additional assumptions of good behavior in terms of 2-dimensional measure, which include having Hausdorff dimension 2. Instead of "uniform local coordinates", one can assume a priori weaker conditions about the geometry and topology. See [DaviS1], [HeiKo1], [Sem1]. For these results, it is important that the dimension be 2, and not larger, because of the way that they rely on the existence of conformal mappings. We shall say a bit more about this in Section 8.

In dimension 3, there are counterexamples, even in the context of good behavior in terms of measure. These examples are based on decompositions of $\mathbf{R}^{3}$, using geometric realizations as in Subsection 7.1. This is discussed in [Sem3]. The absence of a quasisymmetric parameterization in this case is close to a result in $[\mathbf{F r e S}]$, although the setting in [FreS] is different.

Concerning the space $\left(\mathbf{R}^{3} / W\right) \times \mathbf{R}$ considered before, equipped with a nice geometry as from Subsection 7.1 , it is not clear (to my knowledge) whether quasisymmetric parameterizations from $\mathbf{R}^{4}$ exist or not. We saw earlier that bilipschitz mappings do not exist, because of the line in $\left(\mathbf{R}^{3} / W\right) \times \mathbf{R}$ which has to have Hausdorff dimension at least 2 after any 
homeomorphism from $\left(\mathbf{R}^{3} / W\right) \times \mathbf{R}$ onto $\mathbf{R}^{4}$. These considerations of Hausdorff dimension or measure do not by themselves rule out the existence of a quasisymmetric mapping, as they do for bilipschitz mappings. (Compare with [Väi2], for instance.)

Similar remarks apply to double-suspensions of homology spheres. In particular, it is not known (to my knowledge) whether or not quasisymmetric parameterizations exist for them.

\subsection{Examples that are even simpler topologically, but still non- trivial geometrically.}

Let us mention another class of examples, which one can also think of in terms of decompositions (although they are "trivial" in this respect). These examples are based on "Antoine's necklaces", which came up before, in Section 1.

Antoine's necklaces are compact subsets of $\mathbf{R}^{3}$ which are homeomorphic to the usual middle-thirds Cantor set in the real line, but for which there is no global homeomorphism from $\mathbf{R}^{3}$ onto itself which maps these sets into subsets of a line. In dimension 2 this does not happen, as in Chapter 13 of [Moi].

The "wildness" of these sets is manifested in a simple fundamentalgroup property. Namely, the complement of these sets in $\mathbf{R}^{3}$ have nontrivial fundamental group, whereas this would not be true if there were a global homeomorphism from $\mathbf{R}^{3}$ to itself which would take one of these sets to a subset of the line. This last uses the fact that these sets are totally disconnected (i.e., to have simple-connectivity of the complement in $\mathbf{R}^{3}$ if the set were to lie in a line).

Antoine's necklaces are discussed in Chapter 18 of [Moi]. See also p. 71ff in [Dave2]. The basic construction for them can be described in terms of the same kind of "rules" as in Subsection 7.1.

One starts with a solid torus $T$ in $\mathbf{R}^{3}$. Inside this torus one embeds some more tori, which are disjoint, but which form a chain that is "linked" around the hole in the original torus. (See Figure 18.1 on p. 127 of [Moi], or Figure 9.9 on p. 71 of [Dave2].) In each of these smaller tori, one can embed another collection of linking tori, in the same way as for the first solid torus.

One can repeat this indefinitely. In the limit, one gets a Cantor set, which is a necklace of Antoine.

Actually, we should be a little more precise here. In saying that we get a Cantor set in the limit, we are implicitly imagining that the diameters of the solid tori are going to 0 as one proceeds through the generations of the construction. This is easy to arrange, if one uses enough tori in 
the basic rule (linking around the original torus $T$ ). If one uses enough tori, then one can do this in such a way that all of the tori are similar to the initial torus $T$, i.e., can be given as images of $T$ by mappings which are combinations of translations, rotations, and dilations.

One can also consider using a smaller number of tori, and where the embeddings of the new tori (in the original one) are allowed to have some stretching. (Compare with Figure 9.9 on p. 71 of [Dave2].) As an extreme case, the Whitehead continuum corresponds essentially to the same construction as Antoine's necklaces, except that only 1 embedded torus is used in the linking in the original torus T. (See Figure 9.7 on p. 68 of [Dave2].) For this one definitely needs a fair amount of stretching. In Bing doubling, one uses two solid tori, embedded and linked around the hole in $T$ (Figure 3 on p. 357 of [Bin1], Figure 9.1 on p. 63 in [Dave2]). One again needs some stretching, but not as much for the Whitehead continuum.

These cases are different from standard ones for Antoine's necklaces, because when one iterates the basic rule in a straightforward way, the components that one gets at the $n$th generation do not have diameters tending to 0 . For the Whitehead continuum, this is simply unavoidable, and reflects the way that the components are clasped, each one by itself, around the "hole" of the original torus $T$. In the case of Bing doubling, one can rearrange the embeddings at later generations in such a way that the diameters do tend to 0 , even if this might not be true for naive iterations. This was proved by Bing, in [Bin1]. (See also p. 69-70 of [Dave2], and [Bin7].)

Let us imagine that we are using enough small solid tori in the linking around $T$, as in standard constructions for Antoine's necklaces, so that it is clear that the diameters of the components of the sets obtained by repeating the process do go to 0 (i.e., without having to make special rearrangements, or anything like that, as in Bing doubling). In other words, in the limit, one gets a Cantor set in $\mathbf{R}^{3}$, as above. Let us call this Cantor set $A$.

This Cantor set $A$ is wild, in the sense that its complement in $\mathbf{R}^{3}$ is not simply-connected, and there is no global homeomorphism from $\mathbf{R}^{3}$ to itself which takes $A$ to a subset of a line. At the level of decompositions, however, there is not much going on here.

Normally, to get a decomposition from a process like this, one takes the connected components obtained in the limit of the process, together with sets with one element for the rest of the points in $\mathbf{R}^{3}$ (or $\mathbf{R}^{n}$, as the case may be). This was described before, in Subsection 7.1. (See also the discussion at the beginning of Section 9 of [Dave2], on p. 61, 
concerning the notion of a defining sequence.) In the construction that we are considering here, all of the connected components in the end contain only one element each, because of the way that the diameters of the components in finite stages of the "defining sequence" converge to 0 .

In other words, the decomposition that occurs here is automatically "trivial", consisting of one-element sets, one for each point in $\mathbf{R}^{3}$. Taking the quotient does not do anything, and the decomposition space is just $\mathbf{R}^{3}$ again.

There is nothing too complicated about this. It is just something to say explicitly, for the record, so to speak, to be clear about it, especially since it is a situation to which one might normally pay little attention, for being degenerate. (See also the text at the beginning of p. 71 of [Dave2], about this kind of defining sequence and decomposition.)

While there is nothing going on at the level of the decompositions topologically, this is not the case geometrically! One can think of this in the same way as in Subsection 7.1, for making geometric representations of decomposition spaces, with metrics and self-similarity properties for them. In the present situation, one can also work more directly at the level of $\mathbf{R}^{3}$ itself, to get geometries like this.

Here is the basic point. Imagine deforming the geometry of $\mathbf{R}^{3}$, at the level of infinitesimal measurements of distance, as with Riemannian metrics. In the general idea of a decomposition space, one can shrink sets in some $\mathbf{R}^{n}$ which have nonzero diameter to single points. In the present setting, our basic components already are single points, and so there is nothing to do to them. However, one can still shrink the geometry around these points in $\mathbf{R}^{3}$.

In technical terms, one can think of deforming the geometry of $\mathbf{R}^{3}$ by multiplying its standard Riemannian metric by a function. One can take this function to be positive and regular away from the Antoine's necklace, and then vanish on the necklace itself. For instance, one could take the function to be a positive power of the distance to the necklace $A$, so that the Riemannian metric can be written as follows:

$$
d s^{2}=\operatorname{dist}(x, A)^{\alpha} d x^{2} .
$$

This type of metric is discussed in some detail in [Sem4] (although in a slightly different form). The geometry of $\mathbf{R}^{3}$ with this kind of metric behaves a lot like standard Euclidean geometry. One still has basic properties like Ahlfors-regularity of dimension 3, and Sobolev, Poincaré, and isoperimetric inequalities in the new geometry. See [Sem4].

However, with suitable choices of parameters, the metric space that one gets in this way is not bilipschitz equivalent to $\mathbf{R}^{3}$ with the standard 
Euclidean metric. This is because the shrinking of distances around the necklace $A$ can lead to $A$ having Hausdorff dimension less than 1 in the new metric. On the other hand, $\mathbf{R}^{3} \backslash A$ is not simply-connected, and this means that the geometry which has been constructed cannot be bilipschitz equivalent to the standard geometry on $\mathbf{R}^{3}$, because of Theorem 5.2 in Section 5. See [Sem4] for more information. (Concerning the "choice of parameters" here, the main point is to have enough shrinking of distances around $A$ to get the Hausdorff dimension to be less than 1, or something like that. The amount of shrinking needed depends on some of the choices involved in producing the necklace. By using Antoine's necklaces which are sufficiently "thin", it is enough to employ arbitrarily small powers $\alpha$ of the distance to the necklace in (7.4) to get enough shrinking of the metric around the necklace. In any case, one is always free to take the power $\alpha$ to be larger.)

Let us emphasize that in making this kind of construction, the conclusion is that the metric space that one gets is not bilipschitz-equivalent to $\mathbf{R}^{3}$ through any homeomorphism between the two spaces. It is easy to make deformations of the geometry so that the new metric seems to be much different from the old one in the given coordinates, but for which this is not really the case if one is allowed to make a change of variables. For instance, one might deform the standard Euclidean Riemannian metric on $\mathbf{R}^{3}$ by multiplying it by a function that vanishes at a point, like a positive power of the distance to that point. Explicitly, this means

$$
d \widetilde{s}^{2}=|x-p|^{\beta} d x^{2},
$$

where $\beta>0$. In the standard coordinates, this metric and the ordinary one look quite different. However, for this particular type of deformation (as in (7.5)), the two metrics are bilipschitz equivalent, if one allows a nontrivial change of variables. Specifically, one can use changes of variables of the form

$$
f(x)=p+|x-p|^{\beta / 2}(x-p) .
$$

This is not hard to verify.

As a more complicated version of this, one can also make deformations of the standard geometry on $\mathbf{R}^{3}$ of the form

$$
d \widehat{s}^{2}=\operatorname{dist}(x, K)^{\gamma} d x^{2},
$$

where $\gamma>0$ and $K$ is a self-similar Cantor set in $\mathbf{R}^{3}$ which is not wild. In this case one can again get geometries which may look different from the usual one in the given coordinates, but for which there are changes of 
variables which give a bilipschitz equivalence with the standard metric. Compare with Remark 5.28 on p. 390 of [Sem4].

When one makes deformations based on Antoine's necklaces, as above, the linking that goes on can ensure that there is no bilipschitz equivalence between $\mathbf{R}^{3}$ with the new geometry and $\mathbf{R}^{3}$ with the standard geometry. In fact, there will not be a homeomorphism which is Lipschitz (from the new geometry to the standard one), without asking for bilipschitzness. With suitable choices of parameters, it can be impossible to have a homeomorphism like this which is even Hölder continuous of an arbitrary exponent $\delta>0$, given in advance.

Under the conditions in [Sem4], the identity mapping itself on $\mathbf{R}^{3}$ always gives a homeomorphism which is Hölder continuous with some positive exponent. In fact, it is also quasisymmetric, in the sense of [TukV]. Thus, here one gets examples of spaces which are quasisymmetrically equivalent to a Euclidean space, and which are Ahlfors-regular of the correct dimension, but which are not bilipschitz equivalent to a Euclidean space. (Compare with Subsection 7.2.)

\section{Geometric and analytic results about the existence of good coordinates}

In Section 2, we considered the question of whether a nonempty contractable open set in $\mathbf{R}^{n}$ is homeomorphic to the standard open unit ball in $\mathbf{R}^{n}$. When $n=2$ this is true, and it is a standard result in topology.

One can establish this result in 2 dimensions analytically via the Riemann Mapping Theorem. This theorem gives the existence of a conformal mapping from the unit disk in $\mathbf{R}^{2}$ onto any nonempty simplyconnected open set in $\mathbf{R}^{2}$ which is not all of $\mathbf{R}^{2}$. See Chapter 6 of [Ahl1], for instance.

The Riemann Mapping Theorem is of course very important for many aspects of analysis and geometric function theory in $\mathbf{R}^{2} \cong \mathbf{C}$, but it also does a lot at a less special level. From it one not only obtains homeomorphisms from the unit disk in $\mathbf{R}^{2}$ onto any nonempty simply-connected proper open subset of $\mathbf{R}^{2}$, but one gets a way of choosing such homeomorphisms which is fairly canonical. In particular, Riemann mappings are unique modulo a three real-dimensional group of automorphisms of the unit disk (which can be avoided through suitable normalizations), and there are results about the dependence of Riemann mappings on the domains being parameterized.

By comparison, one might try to imagine doing such things without the Riemann mapping, or in other contexts where it is not available. 
In this regard, see $[$ Hat1], [Hat2], $[\mathbf{L a u}],[\mathbf{R a n S}]$, concerning related matters in higher dimensions.

Another fact in dimension 2 is that any smooth Riemannian metric on the 2-sphere $\mathbf{S}^{2}$ is conformally-equivalent to the standard metric. In other words, if $g$ is a smooth Riemannian metric on $\mathbf{S}^{2}$, and if $g_{0}$ denotes the standard metric, then there is a diffeomorphism from $\mathbf{S}^{2}$ onto $\mathbf{S}^{2}$ which converts $g$ into a metric of the form $\lambda g_{0}$, where $\lambda$ is a smooth positive function on $\mathbf{S}^{2}$. There are also local and other versions of this fact, but for the moment let us stick to this formulation.

One way to try to use this theorem is as follows. Suppose that one has a 2-dimensional space which behaves roughly like a 2-dimensional Euclidean space (or sphere) in some ways, and one would like to know whether it can be realized as nearly-Euclidean in more definite ways, through a parameterization which respects the geometry. Let us assume for simplicity that our space is given to us as $\mathbf{S}^{2}$ with a smooth Riemannian metric $g$, but without bounds for the smoothness of $g$. One can then get a conformal diffeomorphism $f:\left(\mathbf{S}^{2}, g_{0}\right) \rightarrow\left(\mathbf{S}^{2}, g\right)$, as in the result mentioned in the preceding paragraph. A priori the behavior of this mapping could be pretty complicated, and one might not know much about it at definite scales. It would be nice to have some bounds for the behavior of $f$, in terms of simple geometric properties of $\left(\mathbf{S}^{2}, g\right)$.

Some results of this type are given in [DaviS1], [HeiKo1], [Sem1]. Specifically, general conditions are given in [DaviS1], [HeiKo1], [Sem1] under which a conformal equivalence $f:\left(\mathbf{S}^{2}, g_{0}\right) \rightarrow\left(\mathbf{S}^{2}, g\right)$ actually gives a quasisymmetric mapping (as in $[\mathbf{T u k V}]$ ), with uniform bounds for the quasisymmetry condition. In other words, these results have the effect of giving uniform bounds for the behavior of $f$ at any location or scale, under suitable conditions on the initial space $\left(\mathbf{S}^{2}, g\right)$, and using the conformality of $f$.

Another use of conformal mappings of an analogous nature is given

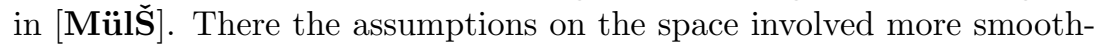
ness - an integral condition on (principal) curvatures, for a surface in some $\mathbf{R}^{n}$ - and the conclusions are also stronger, concerning bilipschitz coordinates. This gave a new approach to results in [Tor1]. See also [Tor2], and the recent and quite different method in $[\mathbf{F u}]$.

More precisely, [Mülš $]$ works with conformal mappings, while [Tor1], [Tor2] and [Fu] obtain bilipschitz coordinates by quite different means. In the context of [DaviS1], [HeiKo1], [Sem1], no other method for getting quasisymmetric or other coordinates with geometric estimates (under similar conditions) is known, at least to my knowledge. 
One might keep in mind that conformality is defined in infinitesimal terms, through the differential of $f$. To go from infinitesimal or very small-scale behavior to estimates at larger scales, one in effect tries to "integrate" the information that one has.

This is a very classical subject for conformal and quasiconformal mappings. A priori, it is rather tricky, because one is not given any information about the conformal factors (like the function $\lambda$ before). Thus one cannot "integrate" directly in a conventional sense. One of the basic methods is that of "extremal length", which deals with the balance between length and area. At any rate, methods like these are highly nonlinear. See $[\mathbf{A h l 2}]$, [Ahl3], [LehV], [Väi1] for more information.

What would happen if one attempted analogous enterprises in higher dimensions? One can begin in the same manner as before. Let $n$ be an integer greater than or equal to 2, and suppose (as a basic scenario) that one has a smooth Riemannian metric $g$ on $\mathbf{S}^{n}$. Let $g_{0}$ denote the standard metric on $\mathbf{S}^{n}$. One might like to know that $\left(\mathbf{S}^{n}, g\right)$ can be parameterized by $\left(\mathbf{S}^{n}, g_{0}\right)$ through a mapping with reasonable properties, and with suitable bounds, under some (hopefully modest) geometric conditions on $\left(\mathbf{S}^{n}, g\right)$. Here, as before, the smoothness of $g$ should be taken in the character of an a priori assumption. One would seek uniform bounds that do not depend on this in a quantitative way. (The bounds would depend on constants in the geometric conditions on $\left(\mathbf{S}^{n}, g\right)$.)

If one has a mapping $f:\left(\mathbf{S}^{n}, g_{0}\right) \rightarrow\left(\mathbf{S}^{n}, g\right)$ which is conformal, or which is quasiconformal (with a bound for its dilatation), then [HeiKo1] provides some natural hypotheses on $\left(\mathbf{S}^{n}, g\right)$ under which one can establish that $f$ is quasisymmetric, and with bounds. In other words, this works for all $n \geq 2$, and not just $n=2$, as above. See [HeiKo2], [HeiKo3] for further results along these lines.

However, when $n>2$, there are no general results about existence of conformal parameterizations for a given space, or quasiconformal parameterizations with uniform bounds for the dilatation. It is simply not true that arbitrary smooth Riemannian metrics admit conformal coordinates, even locally, as they do when $n=2$. Quasiconformal coordinates automatically exist for reasonably-nice metrics, but with the quasiconformal dilatation depending on the metric or on the size of the region being parameterized in a strong way. The issue would be to avoid or reduce that.

One can easily see that the problem is highly overdetermined, in the following sense. A general Riemannian metric in $n$ dimensions is described (locally, say) by $n(n+1) / 2$ real-valued functions of $n$ variables. 
A conformal deformation of the standard metric is defined by 1 realvalued function of $n$ real variables, i.e., for the conformal factor. A general diffeomorphism in $n$ dimensions is described by $n$ real-valued functions of $n$ variables. Thus, allowing for general changes of variables, the metrics which are conformally-equivalent to the standard metric are described by $n+1$ real-valued functions of $n$ real variables. When $n=2$, this is equal to $n(n+1) / 2$, but for $n>2$ one has that $n(n+1) / 2>n+1$.

In fact, one knows that in dimension 3 there are numerous examples of spaces which satisfy geometric conditions analogous to ones that work in dimension 2, but which do not admit quasisymmetric parameterizations. There are also different levels of structure which occur in dimension 3, between basic geometric properties and having quasisymmetric parameterizations, and which would come together in dimension 2. Parts of this are reviewed or discussed in Section 7, especially Subsection 7.2; see $[\mathbf{S e m} 3]$ for more information.

Thus, not only does the method based on conformal and quasiconformal mappings not work in higher dimensions, but some of the basic results that one might hope to get or expect simply are not true, by examples which are pretty concrete.

This is all pretty neat! One has kinds of "parallel tracks", with geometric topology on one side, and aspects of geometry and analysis on the other. A priori, these two tracks can exist independently, even if there are ways in which each can be involved in the other.

Each of these two tracks has special features in low dimensions. This concerns the existence of homeomorphisms with certain properties, for instance. Each has statements and results and machinery which make sense for the given track, and not for the other side, even if there are also some overlaps (as with applications of Riemann mappings).

Each of these two tracks also starts running into trouble in higher dimensions, and at about the same time! The kinds of trouble that they encounter can be rather different a priori, even if there is again significant overlap between them.

\subsection{Special coordinates that one might consider in other di- mensions.}

Let us now briefly consider a couple of things that one might try in higher dimensions on the side of geometry and analysis, in similar veins as above.

One basic approach would be to try to find and use mappings which minimize some kind of "energy". As before, one can consider smooth metrics on smooth manifolds (like $\mathbf{S}^{n}$ ), and try to get parameterizations 
with uniform bounds on their behavior, under modest conditions on the geometry of the spaces. (One can also try to work directly with spaces and metrics that are not smooth.)

A very standard energy functional to consider would be the $L^{2}$ norm of the differential, as with harmonic mappings. In dimension 2 , conformal mappings can be placed in this framework. One can also consider energy functionals based on $L^{p}$ norms of differentials of mappings. This is more complicated in terms of the differential equations that come up, but it can have other advantages. The choice of $p$ as the dimension $n$ has some particularly nice features, for having the energy functional cooperate with the geometry (and analysis). (This is one of the ways that $n=2$ is special; for this one can have both $p=n$ and $p=2$ at the same time!) In particular, the energy becomes invariant under conformal changes in the metric when $p$ is equal to the dimension.

In elasticity theory, one considers more elaborate energy functionals as well. For instance, these might include integral norms of the inverse of the Jacobian of the mapping, in addition to $L^{p}$ norms of the differentials. In other words, the functional can try to limit both the way that the differential becomes large and small, so that it takes into account both stretchings and compressions.

In any case, although there is a lot of work concerning existence and behavior of minimizers for functionals like these, I do not really know of results in dimensions $n \geq 3$ where they can be used to obtain wellbehaved parameterizations of spaces, with bounds, under modest or general geometric conditions. This is especially true in comparison with what one can get in dimension 2 , as discussed before.

In dimension 3, there is another kind of special structure that one might consider. Namely, instead of metrics which are conformal deformations of the standard Euclidean metric, let us consider metrics $g=g_{i, j}$ for which only the diagonal entries $g_{i, i}$ are nonzero.

In this case the diagonal entries are allowed to vary independently. For conformal deformations of the standard Euclidean metric, the offdiagonal entries are zero, and the diagonal entries are all equal.

In dimension 3 , the problem of making a change of variables to put a given metric into diagonal form like this is "determined", in the same way as for conformal deformations of Euclidean metrics in dimension 2. Specifically, one can compute as follows. A general Riemannian metric is described by $n(n+1) / 2$ real-valued functions of $n$ variables, which means 6 real-valued functions of 3 real variables in dimension 3. Metrics with only diagonal nonzero entries are defined by 3 real-valued functions of 
3-real variables, and changes of variables are given by 3 real-valued functions of 3 real variables as well. Thus, allowing for changes of variables, the metrics that can be reduced to diagonal metrics can be described by 6 real-valued functions of 3 real-variables, which is the same as for the total family of Riemannian metrics in this dimension. In dimensions greater than or equal to 4, this would not work, and there would again be too many Riemannian metrics in general compared to diagonal metrics and ways of reducing to them via changes of variables.

Of course this is just an informal "dimension" count, and not a justification for being able to put metrics into diagonal form in dimension 3 . (One should also be careful that there is not significant overlap between changes of variables and diagonal metrics, i.e., so that there was no "overcounting" for the combination of them.) However, it does turn out that one can put metrics in diagonal form (in dimension 3), at least locally. This was established in $[\mathbf{D e T Y}]$ in the case of smooth metrics. There were earlier results in the real-analytic category. (See [DeTY] for more information.)

However, this type of "normal form" does not seem to be as useful for the present type of issue as conformal parameterizations are. As in the case of conformal coordinates, part of the problem is that even if one has such a normal form, one does not a priori know anything about the behavior of the diagonal entries of the metric in this normal form. One would need methods of getting estimates without this information, and only the nature of the normal form. In the context of conformal mappings, one has extremal lengths, conformal capacities, and other conformal and quasiconformal invariants and quasi-invariants. For diagonal metrics, it is not clear what one might do.

A related point is that the analysis of the partial differential equations which permits one to put smooth Riemannian metrics in dimension 3 into diagonal form is roughly "hyperbolic", in the same way that the corresponding differential equations for conformal coordinates in dimension 2 are elliptic. See [DeTY]. This is closely connected to the kind of stability that one has for conformal mappings, and the possibilities for having estimates for them under mild or primitive geometric conditions.

In a way this is all "just fair", and nicely so. With diagonal metrics one does have something analogous to conformal coordinates in dimension 3 . On the other hand, this analogue behaves differently in fundamental ways, including estimates. This is compatible with other aspects of the story as a whole, like the topological and geometric examples that one has in dimension 3 (where homeomorphisms may not exist, with the properties that one might otherwise hope for). 
In any event, this illustrates how analytic and geometric methods seem to behave rather differently in dimensions 3 and higher, compared to the special structure and phenomena which occur in dimension 2 . This is somewhat remarkable in analogy with topological phenomena, which have similar differences between dimensions. With the topology there are both some crossings and overlaps with geometry and analysis, and much that is separate or independent.

On the side of geometry and analysis, let us also note that there are some other special features in low dimensions that we have not mentioned. As a basic example, the large amount of flexibility that one has in making conformal mappings in dimension 2 leads to some possibilities in dimension 3 that are not available in higher dimensions. That is, the large freedom that one has in dimension 2 can sometimes permit one to make more limited constructions in dimension 3, e.g., by starting with submanifolds of dimension 2, and working from there (with extensions, gluings, etc.) These possibilities in dimension 3 can be much more restricted than in dimension 2 , but having them at all can be significantly more than what happens in higher dimensions.

Concerning variational problems, one might also keep in mind the approaches of [DaviS2], [DaviS3] (and some earlier ideas of Morel and Solimini $[$ MoreS $]$ ). For these one does not necessarily work directly with mappings or potential parameterizations of sets, and in particular one may allow sets themselves to be variables in the minimization (rather than mappings between fixed spaces). This broader range can make it easier for the minimizations to lead to useful conclusions about geometric structure and complexity, under natural and modest conditions. In particular, one can get substantial "partial parameterizations", as with uniform rectifiability conditions.

These approaches are also nicely compatible with the trouble that one knows can occur, related to topology and homeomorphisms (and in geometrically moderate situations, as in Subsections 7.2 and 7.3, and $[$ Sem3], [Sem4]).

Finally, while we have mentioned a lot about the special phenomena that can occur in dimension 2, and what happens in higher dimensions, we should also not forget about dimension 1 . This is even more special than dimension 2. This is a familiar theme in geometric topology, for the ways that one can recognize and parameterize curves. In geometry and analysis, one can look for parameterizations with bounds, and these are often constructible. 
A fundamental point along these lines is the ability to make parameterizations by arclength, for curves of locally finite length. More generally, one can use parameterizations adapted to other measures (rather than length), when they are around.

Arclength parameterizations provide a very robust and useful way for obtaining parameterizations in dimension 1 with good behavior and bounds. In dimension 1, simple conditions in terms of mass can often be immediately "integrated" to get well-behaved parameterizations, in ways that are not available (or do not work nearly as well) in higher dimensions, even in dimension 2.

To put the matter in more concrete terms, in dimension 1 one can often make parameterizations, or approximate parameterizations, simply by ordering points in a good way. This does not work in higher dimensions. Once one has the ordering, one can regularize the geometry by parameterizing according to arclength, or some other measure (as appropriate).

For another version of this, in connection with quasisymmetric mappings, see Section 4 of [TukV].

In differential-geometric language, one might say that dimension 1 is special for the way that one can make isometries between spaces, through arclength parameterizations. This no longer works in dimension 2, but one has conformal coordinates there. Neither of these are generally available in higher dimensions. In higher dimensions one has less special structure for getting the existence in general of well-behaved parameterizations, and then the kinds and ranges of geometric and topological phenomena which can exist open up in a large way.

\section{Nonlinear similarity: Another class of examples}

A very nice and concrete situation in which issues of existence and behavior of homeomorphisms can come up is that of "nonlinear similarity". Specifically, it is possible to have linear mappings $A, B$ on $\mathbf{R}^{n}$ which are conjugate to each other by homeomorphisms from $\mathbf{R}^{n}$ onto itself -i.e., $B=h \circ A \circ h^{-1}$, where $h$ is a homeomorphism of $\mathbf{R}^{n}$ onto itself - and which are not conjugate by linear mappings!

Examples of this were given in $[\mathbf{C a p S 1}]$, [CapS2]. For related matters, including other examples, conditions under which one can deduce linear equivalence, and more on the behavior of conjugating homeomorphisms when a linear equivalence does not exist, see [CapS3], [CapS4], [CapS5], [CapS+], [CapS*], [HamP1], [HamP2], [HsiP1], [HsiP2], 
[KuiR], [MadR1], [MadR2], [Mio], [Rha1], [Rha2], [RotW], [Wei1], [Wei2], [Wilk].

\section{Doing pretty well with spaces which may not have nice coordinates}

If one has a topological or metric space (or whatever) which has nice coordinates, then that can be pretty good. However, there is a lot that one can do without having coordinates.

Let us begin with some basic conditions. Let $M$ be a topological space which is compact, Hausdorff, and metrizable. We shall assume that $M$ has finite topological dimension, in the sense of [HurW]. In these circumstances, this is equivalent to saying that $M$ is homeomorphic to a subset of some $\mathbf{R}^{n}$. (See $[\mathbf{H u r W}]$.)

For simplicity, let us assume that $M$ is a compact subset of some $\mathbf{R}^{n}$. It is also convenient to ask that $M$ be locally contractable. This means that for each point $p \in M$, and each neighborhood $U$ of $p$ in $M$, there is a smaller neighborhood $W \subseteq U$ of $p$ in $M$ such that $W$ can be contracted to a point in $U$.

As a class of examples, finite polyhedra are locally contractable. Finite polyhedra make a nice special case to consider throughout this section, and we shall return to it several times.

For another class of examples, one has cell-like quotients of topological manifolds (and of some locally contractable spaces more generally), at least when the quotient spaces have finite topological dimension. See Corollary 12B on p. 129 of [Dave2].

As a general fact about local contractability, let us note the following.

Proposition 10.1. Let $M$ be a compact subset of some $\mathbf{R}^{n}$. Then $M$ is locally contractable if and only if there is a set $V \subseteq \mathbf{R}^{n}$ which contains $M$ in its interior, and a continuous mapping $r: V \rightarrow M$ which is a retract, i.e., $r(w)=w$ for all $w \in V$.

This is a fairly standard observation. The "if" part is an easy consequence of the local contractability of $\mathbf{R}^{n}$ (through linear mappings). Specifically, to get local contractions inside of $M$, one makes standard linear contractions in $\mathbf{R}^{n}$, which normally do not stay inside $M$, and then one applies the retraction to keep the contractions inside $M$.

For the converse, one can begin by defining $r$ on a discrete and reasonably-thick set of points outside $M$, but near $M$. For a point $w$ in such a set, one could choose $r(w) \in M$ so that it lies as close to $w$ as possible (among points in $M$ ), or is at least approximately like this. To fill 
in $r$ in the areas around these discrete points, one can make extensions first to edges, then 2-dimensional faces, and so on, up to dimension $n$. To make these extensions, one uses local contractability of $M$. It is also important that the local extensions do not go to far from the selections already made, so that $r: V \rightarrow M$ will be continuous in the end, and this one can get from the local contractability.

The notion of "Whitney decompositions", as in Chapter VI of [Ste], is helpful for this kind of argument. It gives a way of decomposing $\mathbf{R}^{n} \backslash M$ into cubes with disjoint interiors, and some other useful properties. (In particular, this kind of decomposition can be helpful for keeping track of bounds, if one should wish to do so.) One can use the vertices of these cubes for the discrete set in the complement of $M$ mentioned above.

See also [Dave2] concerning Proposition 10.1, especially p. 117ff.

Let us return now to the general story. Suppose that $M$ is a compact subset of $\mathbf{R}^{n}$, and that $M$ is locally contractable. Let $r: V \rightarrow M$ be a continuous retraction on $M$, as in Proposition 10.1. Thus $V$ contains $M$ in its interior. By replacing $V$ by a slightly smaller subset, if necessary, we may assume that $V$ is compact, and in fact that it is a finite union of dyadic cubes in $\mathbf{R}^{n}$. (A dyadic cube in $\mathbf{R}^{n}$ is a cube which is a Cartesian product of intervals of the form $\left[j_{i} 2^{-k},\left(j_{i}+1\right) 2^{-k}\right], i=1,2, \ldots, n$, where the $j_{i}$ 's and $k$ are integers.)

This type of choice for $V$ is convenient for having nice properties in terms of homology and cohomology. In particular, $V$ is then a finite complex. The inclusion of $M$ into $V$, and the mapping $r: V \rightarrow M$, induce mappings between the homology and cohomology of $M$ and $V$. If $\iota: M \rightarrow V$ denotes the mapping coming from inclusion, then $r \circ \iota: M \rightarrow$ $M$ is the identity mapping, and thus it induces the identity mapping on the homology and cohomology of $M$. Using this, one can see that the mapping from the homology of $M$ into the homology of $V$ induced by $\iota$ is an injection (in addition to being a group homomorphism, as usual), and that the mapping from the homology of $V$ to the homology of $M$ induced by $r$ is a surjection. This follows from standard properties of homology and mappings, as in [Mas]. Similarly, $r$ induces a mapping from cohomology of $M$ to cohomology of $V$ which is injective, and $\iota$ induces a mapping from cohomology of $V$ into cohomology of $M$ which is surjective.

This provides a simple way in which the algebraic topology of $M$ can be "bounded", under the type of assumptions on $M$ that we are making. (There are more refined things that one can also do, but we shall not worry about this here.) Local contractability, and the existence of a retraction as in Proposition 10.1, are also nice for making it clear and 
easy to work with continuous mappings into $M$. In particular, one can get continuous mappings into $M$ from continuous mappings into $V$, when one has a retraction $r: V \rightarrow M$, as above. This is as opposed to standard examples like the closure of the graph of $\sin (1 / x), x \in[-1,1] \backslash\{0\}$. (This set is connected but not arcwise connected.)

Now let us consider the following stronger conditions on $M$.

Definition 10.2 (Generalized $k$-Manifolds). Let $M$ be a compact subset of $\mathbf{R}^{n}$ which is locally contractable. Then $M$ is a generalized $k$-manifold if for every point $z \in M$, the relative homology $H_{j}(M, M \backslash\{z\})$ is the same (up to isomorphism) as the relative homology $H_{j}\left(\mathbf{R}^{k}, \mathbf{R}^{k} \backslash\{0\}\right.$ ) for each $j$.

We are implicitly working with homology defined over the integers here, and there are analogous notions with respect to other coefficient groups (like rational numbers, for instance). One may also wish to use weaker conditions than local contractability (as in [Bre2], [Wild]). There are other natural variations for this concept.

If $M$ is a finite polyhedron, then the property of being a generalized manifold is equivalent to asking that the links of $M$ be homology spheres of the right dimension (i.e., with the same homology as a standard sphere, up to isomorphism).

Another class of examples comes from taking quotients of compact topological manifolds by cell-like decompositions (Sections 4 and 6), at least when the quotient space has finite topological dimension. See Corollary 1A on p. 191 of [Dave2] (and Corollary 12B on p. 129 there), and compare also with Theorem 16.33 on p. 389 of [Bre2], and [Fer].

As usual, dimensions 1 and 2 are special for generalized manifolds, which are then topological manifolds. See [Wild], Theorem 16.32 on p. 388 of [Bre2], and the introduction to [Fer].

For more on ways that generalized manifolds can arise, see [Bor2], [Bre2], [Bry+], [Bry*], [Dave2], [Fer], [Wei2] (and the references therein). A related topic is the "recognition problem", for determining when a topological space is a topological manifold. Some references for this include $[$ Bry+], [Bry*], [Can1], [Can2], [Can3], [Dave2], [Edw2], [Fer], [Wei2].

What are some properties of generalized manifolds? In what ways might they be like manifolds?

A fundamental point is that Poincaré duality (and other duality theorems for manifolds) also work for generalized manifolds. See [Bor1], [Bor2], [Bre2], [Wild] and p. 277-278 of [Spa]. This is pretty good, 
since Poincaré duality is such a fundamental aspect of manifolds. (See $[$ BotT], [Bre1], [Mas], [MilS], [Spa], for instance.)

A more involved fact is that rational Pontrjagin classes can be defined for generalized manifolds. (See the introduction to $[\mathrm{Bry}+]$.)

For smooth manifolds, the definition of the Pontrjagin classes is classical. (See $[$ BotT], [MilS].) More precisely, one can define Pontrjagin classes for vector bundles in general, and then apply this to the tangent bundle of a smooth manifold to get the Pontrjagin classes of a manifold. As integral cohomology classes, the Pontrjagin classes are preserved by diffeomorphisms between smooth manifolds, but not, in general, by homeomorphisms. However, a famous theorem of Novikov is that the Pontrjagin classes of smooth manifolds are preserved as rational cohomology classes by homeomorphisms in general. Further developments lead to the definition of rational characteristic classes on more general spaces.

For finite polyhedra, there is an earlier treatment of rational Pontrjagin classes, which goes back to work of Thom and Rohlin and Schwarz. See Section 20 of [MilS]. More precisely, this gives a procedure by which to define rational Pontrjagin classes for finite polyhedra which are generalized manifolds, and which is invariant under piecewise-linear equivalence. (For this, the generalized-manifold condition can be given in terms of rational coefficients for the homology groups.) If one starts with a smooth manifold, then there it can be converted to a piecewise-linear manifold (unique up to equivalence) by earlier results, and the classical rational Pontrjagin classes for the smooth manifold are the same as the ones that are obtained by the procedure for polyhedral spaces. See [MilS] for more information.

\section{References}

[Ahl1] L. V. Ahlfors, "Complex analysis", An introduction to the theory of analytic functions of one complex variable, Third ed., International Series in Pure and Applied Mathematics, McGraw-Hill Book Co., New York, 1978.

[Ahl2] L. V. Ahlfors, "Lectures on quasiconformal mappings", Van Nostrand Mathematical Studies 10, D. Van Nostrand Co., Inc., Toronto, Ont.-New York-London, 1966.

[Ahl3] L. V. Ahlfors, "Conformal invariants: topics in geometric function theory", McGraw-Hill Series in Higher Mathematics, McGraw-Hill Book Co., New York, 1973. 
[AleV] P. Alestalo and J. VÄısÄLÄ, Quasisymmetric embeddings of products of cells into the euclidean space, Ann. Acad. Sci. Fenn. Ser. A I Math. 19(2) (1994), 375-392.

[AndC] J. J. Andrews And M. L. CuRTis, $n$-space modulo an arc, Ann. of Math. (2) 75 (1962), 1-7.

[AndR] J. J. Andrews And L. Rubin, Some spaces whose product with $E^{1}$ is $E^{4}$, Bull. Amer. Math. Soc. 71 (1965), 675-677.

[Arm] S. Armentrout, Bing's dogbone space is not strongly locally simply connected, in "Topics in low-dimensional topology in honor of Steve Armentrout", (A. Banyaga, H. Movahedi-Lankarani and R. Wells, eds.), World Scientific, 1999, pp. 33-64.

[Bin1] R. H. BING, A homeomorphism between the 3-sphere and the sum of two solid horned spheres, Ann. of Math. (2) 56 (1952), 354-362.

[Bin2] R. H. Bing, A decomposition of $E^{3}$ into points and tame arcs such that the decomposition space is topologically different from $E^{3}$, Ann. of Math. (2) 65 (1957), 484-500.

[Bin3] R. H. BiNG, The cartesian product of a certain non-manifold and a line is $E_{4}$, Bull. Amer. Math. Soc. 64 (1958), 82-84.

[Bin4] R. H. Bing, The cartesian product of a certain nonmanifold and a line is $E^{4}$, Ann. of Math. (2) 70 (1959), 399-412.

[Bin5] R. H. Bing, A surface is tame if its complement is 1-ULC, Trans. Amer. Math. Soc. 101 (1961), 294-305.

[Bin6] R. H. Bing, "The geometric topology of 3-manifolds", American Mathematical Society Colloquium Publications 40, American Mathematical Society, Providence, R.I., 1983.

[Bin7] R. H. Bing, Shrinking without lengthening, Topology 27(4) (1988), 487-493.

[Bin8] R. H. Bing, "Collected papers. Vol. 1, 2", edited and with a preface by Sukhjit Singh, Steve Armentrout and Robert J. Daverman, American Mathematical Society, Providence, RI, 1988.

[Bla] W. A. Blankinship, Generalization of a construction of Antoine, Ann. of Math. (2) 53 (1951), 276-297.

[Bor1] A. Borel, The Poincaré duality in generalized manifolds, Michigan Math. J. 4 (1957), 227-239.

[Bor2] A. BoREL, "Seminar on transformation groups", with contributions by G. Bredon, E. E. Floyd, D. Montgomery, R. Palais, Annals of Mathematics Studies 46, Princeton University Press, Princeton, N.J., 1960. 
[BotT] R. BotT AND L. W. TU, "Differential forms in algebraic topology", Graduate Texts in Mathematics 82, SpringerVerlag, New York, 1982.

[Bre1] G. E. Bredon, "Topology and geometry", Graduate Texts in Mathematics 139, Springer-Verlag, New York, 1993.

[Bre2] G. E. Bredon, "Sheaf theory", Second edition, Graduate Texts in Mathematics 170, Springer-Verlag, New York, 1997.

[BroLL] W. Browder, J. Levine and G. R. Livesay, Finding a boundary for an open manifold, Amer. J. Math. 87 (1965), $1017-1028$.

[Brow1] M. Brown, A proof of the generalized Schoenflies theorem, Bull. Amer. Math. Soc. 66 (1960), 74-76.

[Brow2] M. BRown, Locally flat imbeddings of topological manifolds, Ann. of Math. (2) 75 (1962), 331-341.

[Bry1] J. L. Bryant, Euclidean space modulo a cell, Fund. Math. 63 (1968), 43-51.

[Bry2] J. L. BRYANT, Euclidean $n$-space modulo an $(n-1)$-cell, Trans. Amer. Math. Soc. 179 (1973), 181-192.

[Bry+] J. L. Bryant, S. C. Ferry, W. Mio And S. WeinBerGer, Topology of homology manifolds, Bull. Amer. Math. Soc. (N.S.) 28(2) (1993), 324-328.

[Bry*] J. L. Bryant, S. C. Ferry, W. Mio and S. Weinberger, Topology of homology manifolds, Ann. of Math. (2) 143(3) (1996), 435-467.

[BryS] J. L. BRYANT AND C. L. SEEBECK, III, Locally nice embeddings in codimension three, Quart. J. Math. Oxford Ser. (2) 21 (1970), 265-272.

[Bur] C. E. Burgess, Embeddings of surfaces in Euclidean threespace, Bull. Amer. Math. Soc. 81(5) (1975), 795-818.

[BurC] C. E. Burgess AND J. W. CANnON, Embeddings of surfaces in $E^{3}$, Rocky Mountain J. Math. 1(2) (1971), 259-344.

[Can1] J. W. CANnON, The characterization of topological manifolds of dimension $n \geq 5$, in: "Proceedings of the International Congress of Mathematicians" (Helsinki, 1978), Acad. Sci. Fennica., Helsinki, 1980, pp. 449-454.

[Can2] J. W. CANnON, The recognition problem: what is a topological manifold?, Bull. Amer. Math. Soc. 84(5) (1978), 832-866.

[Can3] J. W. Cannon, Shrinking cell-like decompositions of manifolds. Codimension three, Ann. of Math. (2) 110(1) (1979), $83-112$ 
[CapS1] S. E. Cappell and J. L. Shaneson, Nonlinear similarity of matrices, Bull. Amer. Math. Soc. (N.S.) 1(6) (1979), 899-902.

[CapS2] S. E. Cappell and J. L. Shaneson, Nonlinear similarity, Ann. of Math. (2) 113(2) (1981), 315-355.

[CapS3] S. E. Cappell and J. L. Shaneson, The topological rationality of linear representations, Inst. Hautes Études Sci. Publ. Math. (56) (1982), 101-128.

[CapS4] S. E. Cappell ANd J. L. Shaneson, Nonlinear similarity and differentiability, Comm. Pure Appl. Math. 38(6) (1985), 697-706.

[CapS5] S. E. Cappell and J. L. Shaneson, Non-linear similarity and linear similarity are equivariant below dimension 6 , in " $\mathrm{Tel}$ Aviv Topology Conference: Rothenberg Festschrift (1998)", Contemp. Math. 231, Amer. Math. Soc., Providence, RI, 1999, pp. 59-66.

[CapS+] S. E. Cappell, J. L. Shaneson, M. Steinberger and J. E. West, Nonlinear similarity begins in dimension six, Amer. J. Math. 111(5) (1989), 717-752.

[CapS*] S. E. Cappell, J. L. Shaneson, M. Steinberger, S. Weinberger and J. E. West, The classification of nonlinear similarities over $Z_{2^{r}}$, Bull. Amer. Math. Soc. (N.S.) 22(1) (1990), 51-57.

[Con] R. Connelly, A new proof of Brown's collaring theorem, Proc. Amer. Math. Soc. 27 (1971), 180-182.

[Dave1] R. J. Daverman, Embeddings of $(n-1)$-spheres in Euclidean n-space, Bull. Amer. Math. Soc. 84(3) (1978), 377-405.

[Dave2] R. J. Daverman, "Decompositions of manifolds", Pure and Applied Mathematics 124, Academic Press Inc., Orlando, Fla., 1986.

[DaviS1] G. David and S. Semmes, Quantitative rectifiability and Lipschitz mappings, Trans. Amer. Math. Soc. 337(2) (1993), 855-889.

[DaviS2] G. DAvid and S. Semmes, Quasiminimal surfaces of codimension 1 and John domains, Pacific J. Math. 183(2) (1998), 213-277.

[DaviS3] G. DAvid and S. Semmes, Uniform rectifiability and quasiminimizing sets of arbitrary codimension, Mem. Amer. Math. Soc. 144(687) (2000).

[DeTY] D. M. DeTurck And D. YAng, Existence of elastic deformations with prescribed principal strains and triply orthogonal systems, Duke Math. J. 51(2) (1984), 243-260. 
[Dra] A. N. Dranishnikov, On a problem of P. S. Aleksandrov, Mat. Sb. (N.S.) 135(177)(4) (1988), 551-557, 560; English translation in Math. USSR-Sb. 63(2) (1989), 539-545.

[Edw1] R. D. Edwards, Demension theory. I, in "Geometric topology" (Proc. Conf., Park City, Utah, 1974), Lecture Notes in Math. 438, Springer, Berlin, 1975, pp. 195-211.

[Edw2] R. D. EDWARDS, The topology of manifolds and cell-like maps, in: "Proceedings of the International Congress of Mathematicians" (Helsinki, 1978), Acad. Sci. Fennica., Helsinki, 1980, pp. $111-127$.

[Fal] K. J. FALCONER, "The geometry of fractal sets", Cambridge Tracts in Mathematics 85, Cambridge University Press, Cambridge-New York, 1986.

[Fed] H. FEDERER, "Geometric measure theory", Die Grundlehren der mathematischen Wissenschaften 153, Springer-Verlag New York Inc., New York, 1969.

[Fer] S. C. FERRY, Controlled topology and the characterization of manifolds, Amer. Math. Soc. (to appear).

[Fla] H. Flanders, "Differential forms wih applications to the physical sciences", Academic Press, New York-London, 1963.

[Fre] M. H. Freedman, The topology of four-dimensional manifolds, J. Differential Geom. 17(3) (1982), 357-453.

[FreQ] M. H. Freedman and F. Quinn, "Topology of 4-manifolds", Princeton Mathematical Series 39, Princeton University Press, Princeton, NJ, 1990.

[FreS] M. H. Freedman and R. Skora, Strange actions of groups on spheres, J. Differential Geom. 25(1) (1987), 75-98.

[Fu] J. H. G. FU, Bi-lipschitz rough normal coordinates for surfaces with an $L^{1}$ curvature bound, Indiana Univ. Math. J. 47(2) (1998), 439-453.

[Geh1] F. W. GeHRING, Extension theorems for quasiconformal mappings in $n$-space, in: "Proc. Internat. Congr. Math." (Moscow, 1966), Izdat. "Mir", Moscow, 1968, pp. 313-318.

[Geh2] F. W. Gehring, The Hausdorff measure of sets which link in euclidean space, in: "Contributions to analysis" (a collection of papers dedicated to Lipman Bers), Academic Press, New York, 1974, pp. 159-167.

[HamP1] I. Hambleton and E. K. Pedersen, Non-linear similarity revisited, in: "Prospects in topology" (Princeton, NJ, 1994), Ann. of Math. Stud. 138, Princeton Univ. Press, Princeton, NJ, 1995, pp. 157-174. 
[HamP2] I. Hambleton and E. K. Pedersen, Topological equivalence of linear representations for cyclic groups, Preprint.

[Hat1] A. E. HATChER, Linearization in 3-dimensional topology, in: "Proceedings of the International Congress of Mathematicians" (Helsinki, 1978), Acad. Sci. Fennica., Helsinki, 1980, pp. $463-468$.

[Hat2] A. E. Hatcher, A proof of a Smale conjecture, $\operatorname{Diff}\left(S^{3}\right) \simeq$ $\mathrm{O}(4)$, Ann. of Math. (2) 117(3) (1983), 553-607.

[HeiKo1] J. Heinonen and P. Koskela, Definitions of quasiconformality, Invent. Math. 120(1) (1995), 61-79.

[HeiKo2] J. Heinonen and P. Koskela, From local to global in quasiconformal structures, Proc. Nat. Acad. Sci. U.S.A. 93(2) (1996), 554-556.

[HeiKo3] J. Heinonen AND P. Koskela, Quasiconformal maps in metric spaces with controlled geometry, Acta Math. 181(1) (1998), $1-61$.

[HeiS] J. Heinonen and S. Semmes, Thirty-three yes or no questions about mappings, measures, and metrics, Conform. Geom. Dyn. 1 (1997), 1-12 (electronic).

[HsiP1] W. C. Hsiang And W. PARdon, Orthogonal transformations for which topological equivalence implies linear equivalence, Bull. Amer. Math. Soc. (N.S.) 6(3) (1982), 459-461.

[HsiP2] W. C. Hsiang and W. PARdon, When are topologically equivalent orthogonal transformations linearly equivalent?, Invent. Math. 68(2) (1982), 375-316.

[HurW] W. HuRewicz AND H. Wallman, "Dimension theory", Princeton Mathematical Series 4, Princeton University Press, Princeton, NJ, 1941.

[Ker1] M. A. Kervaire, Smooth homology spheres and their fundamental groups, Trans. Amer. Math. Soc. 144 (1969), 67-72.

[Ker2] M. A. KervaIRe, "Lectures on the theorem of Browder and Novikov and Siebenmann's thesis", Notes by K. Varadarajan, Lectures in Mathematics 46, Tata Institute of Fundamental Research, Bombay, 1969.

[KerM] M. A. Kervaire and J. W. Milnor, Groups of homotopy spheres. I, Ann. of Math. (2) 77 (1963), 504-537.

[Kir] R. C. KIRBY, "The topology of 4-manifolds", Lecture Notes in Mathematics 1374, Springer-Verlag, Berlin-New York, 1989.

[KirS] R. C. Kirby and L. C. Siebenmann, "Foundational essays on topological manifolds, smoothings, and triangulations", with 
notes by John Milnor and Michael Atiyah, Annals of Mathematics Studies 88, Princeton University Press, Princeton, N.J., University of Tokyo Press, Tokyo, 1977.

[KuiR] N. H. Kuiper And J. W. Robbin, Topological classification of linear endomorphisms, Invent. Math. 19 (1973), 83-106.

[Lau] F. LaudenBach, Les 2-sphères de $R^{3}$ vues par A. Hatcher et la conjecture de Smale Diff $\left(S^{3}\right) \sim \mathrm{O}(4)$, Seminar Bourbaki, Vol. 1983/84, Astérisque 121-122 (1985), 279-293.

[LehV] O. Lehto AND K. I. ViRTANen, "Quasiconformal mappings in the plane", Second edition, Translated from the German by K. W. Lucas, Die Grundlehren der mathematischen Wissenschaften 126, Springer-Verlag, New York, 1973.

[LuuV] J. LuUKKAINEN AND J. VÄIsÄL ̈̈, Elements of Lipschitz topology, Ann. Acad. Sci. Fenn. Ser. A I Math. 3(1) (1977), 85-122.

[MadR1] I. Madsen And M. RothenberG, Classifying $G$ spheres, Bull. Amer. Math. Soc. (N.S.) 7(1) (1982), 223-226.

[MadR2] I. MAdsen And M. RothenberG, On the classification of $G$-spheres. I: equivariant transversality, Acta Math. 160(1-2) (1988), 65-104.

[MarRV] O. Martio, S. Rickman And J. VÄıs̈̈LÄ, Topological and metric properties of quasiregular mappings, Ann. Acad. Sci. Fenn. Ser. A I 488 (1971), 1-31.

[Mas] W. S. MASSEY, "A basic course in algebraic topology", Graduate Texts in Mathematics 127, Springer-Verlag, New York, 1991.

[Mat] P. Mattila, "Geometry of sets and measures in Euclidean spaces", Fractals and rectifiability, Cambridge Studies in Advanced Mathematics 44, Cambridge University Press, Cambridge, 1995.

[Maz] B. Mazur, On embeddings of spheres, Bull. Amer. Math. Soc. 65 (1959), 59-65.

[McM] D. R. McMillan, JR., A criterion for cellularity in a manifold, Ann. of Math. (2) 79 (1964), 327-337.

[McMZ] D. R. McMillan, JR. And E. C. Zeeman, On contractible open manifolds, Proc. Cambridge Philos. Soc. 58 (1962), $221-224$.

[Mil] J. W. Milnor, On manifolds homeomorphic to the 7-sphere, Ann. of Math. (2) 64 (1956), 399-405.

[MilS] J. W. Milnor and J. D. StashefF, "Characteristic classes", Annals of Mathematics Studies 76, Princeton University Press, Princeton, N.J., University of Tokyo Press, Tokyo, 1974. 
[Mio] W. MıO, Nonlinearly equivalent representations of quaternionic 2-groups, Trans. Amer. Math. Soc. 315(1) (1989), 305-321.

[Moi] E. E. MoISE, "Geometric topology in dimensions 2 and 3", Graduate Texts in Mathematics 47, Springer-Verlag, New York-Heidelberg, 1977.

[Moo] R. L. Moore, Concerning upper semi-continuous collections of continua, Trans. Amer. Math. Soc. 27(4) (1925), 416-428.

[MoreS] J.-M. Morel AND S. Solimini, "Variational methods in image segmentation", with seven image processing experiments, Progress in Nonlinear Differential Equations and their Applications 14, Birkhäuser Boston, Inc., Boston, MA, 1995.

[Mors] M. Morse, A reduction of the Schoenflies extension problem, Bull. Amer. Math. Soc. 66 (1960), 113-115.

[MülŠ] S. MÜlLeR AND V. ŠverÁk, On surfaces of finite total curvature, J. Differential Geom. 42(2) (1995), 229-258.

[Qui1] F. Quinn, Ends of maps and applications, Bull. Amer. Math. Soc. (N.S.) 1(1) (1979), 270-272.

[Qui2] F. Quinn, Ends of maps. I, Ann. of Math. (2) 110(2) (1979), 275-331.

[Qui3] F. Quinn, Ends of maps. III. Dimensions 4 and 5, J. Differential Geom. 17(3) (1982), 503-521.

[Qui4] F. Quinn, Local algebraic topology, Notices Amer. Math. Soc. 33(6) (1986), 895-899.

[RanS] D. Randall and P. A. Schweitzer, On foliations, concordance spaces, and the Smale conjectures, in "Differential topology, foliations, and group actions" (Rio de Janeiro, 1992), Contemp. Math. 161, Amer. Math. Soc., Providende, RI, 1994, pp. 235-258.

[Rha1] G. DE RHAM, Reidemeister's torsion invariant and rotations of $S^{n}$, in: "Differential Analysis", Bombay Colloq., Oxford Univ. Press, London, 1964.

[Rha2] G. DE Rham, Homéomorphie des rotations de $S^{m}$. Évolution d'un problème, souvenirs, Enseign. Math. (2) 27(3-4) (1981), $177-184$.

[RotW] M. Rothenberg And S. Weinberger, Group actions and equivariant Lipschitz analysis, Bull. Amer. Math. Soc. (N.S.) 17(1) (1987), 109-112.

[Rus1] T. B. Rushing, "Topological embeddings", Pure and Applied Mathematics 52, Academic Press, New York-London, 1973. 
[Rus2] T. B. Rushing, Hausdorff dimension of wild fractals, Trans. Amer. Math. Soc. 334(2) (1992), 597-613.

[Sem1] S. Semmes, Chord-arc surfaces with small constant. II: Good parameterizations, Adv. Math. 88(2) (1991), 170-199.

[Sem2] S. SEmmes, Finding structure in sets with little smoothness, in: "Proceedings of the International Congress of Mathematicians, Vol. 2", (Zürich, 1994), Birkhäuser, Basel, 1995, pp. $875-885$.

[Sem3] S. Semmes, Good metric spaces without good parameterizations, Rev. Mat. Iberoamericana 12(1) (1996), 187-275.

[Sem4] S. Semmes, On the nonexistence of bi-Lipschitz parameterizations and geometric problems about $A_{\infty}$-weights, Rev. Mat. Iberoamericana 12(2) (1996), 337-410.

[Sie] L. C. Siebenmann, The obstruction to finding a boundary for an open manifold of dimension greater than 5, Ph. D. Thesis, Princeton University (1965).

[SieS] L. C. Siebenmann And D. Sullivan, On complexes that are Lipschitz manifolds, in: "Geometric topology" (Proc. Georgia Topology Conf., Athens, Ga., 1977), Academic Press, New York-London, 1979, pp. 503-525.

[Spa] E. H. Spanier, "Algebraic topology", McGraw-Hill Book Co., New York-Toronto, Ont.-London, 1966.

[Sta] J. Stallings, The piecewise-linear structure of Euclidean space, Proc. Cambridge Philos. Soc. 58 (1962), 481-488.

[Ste] E. M. STEIN, "Singular integrals and differentiability properties of functions", Princeton Mathematical Series 30, Princeton University Press, Princeton, N.J., 1970.

[Sul] D. Sullivan, Hyperbolic geometry and homeomorphisms, in: "Geometric Topology" (Proc. Georgia Topology Conf., Athens, Ga., 1977), Academic Press, New York-London, 1979, pp. $543-555$.

[Tor1] T. Toro, Surfaces with generalized second fundamental form in $L^{2}$ are Lipschitz manifolds, J. Differential Geom. 39(1) (1994), 65-101.

[Tor2] T. Toro, Geometric conditions and existence of bi-Lipschitz parameterizations, Duke Math. J. 77(1) (1995), 193-227.

[Tuk] P. TukiA, A quasiconformal group not isomorphic to a Möbius group, Ann. Acad. Sci. Fenn. Ser. A I Math. 6(1) (1981), 149-160. 
[TukV] P. TUKIA AND J. VÄIsÄLÄ, Quasisymmetric embeddings of metric spaces, Ann. Acad. Sci. Fenn. Ser. A I Math. 5(1) (1980), 97-114.

[Väi1] J. VÄISÄLÄ, "Lectures on n-dimensional quasiconformal mappings", Lecture Notes in Mathematics 229, Springer-Verlag, Berlin-New York, 1971.

[Väi2] J. VÄIsÄL̈̈, Quasisymmetric embeddings in Euclidean spaces, Trans. Amer. Math. Soc. 264(2) (1981), 191-204.

[Väi3] J. VÄIsÄL ̈̈, Quasisymmetric maps of products of curves into the plane, Rev. Roumaine Math. Pures Appl. 33(1-2) (1988), $147-156$.

[Wei1] S. Weinberger, The Lipschitz rationality of linear representations, Comm. Pure Appl. Math. 40(5) (1987), 609-610.

[Wei2] S. WEINBERGER, "The topological classification of stratified spaces", Chicago Lectures in Mathematics. University of Chicago Press, Chicago, IL, 1994.

[Whit] J. H. C. WhiteheAD, A certain open manifold whose group is unity, Quart. J. Math. Oxford Ser. (2) 6 (1935), 268-279.

[Wild] R. L. WiLder, "Topology of manifolds", American Mathematical Society Colloquium Publications 32, American Mathematical Society, New York, N. Y., 1949.

[Wilk] J. B. WILkER, Topologically equivalent $n$-dimensional isometries, Topology Appl. 15(2) (1983), 211-221.

Department of Mathematics

Rice University

Box 1892

Houston TX 77251-1892

U.S.A.

E-mail address: semmes@math.rice.edu 